\title{
Coherence of Multiscale Features for Enhancement of Digital Mammograms
}

\author{
Chun-Ming Chang and Andrew Laine
}

\begin{abstract}
Mammograms depict most of the significant changes in breast disease. The primary radiographic signs of cancer are related to tumor mass, density, size, borders, and shape, and local distribution of calcifications. We show that each of these features can be well described by coherence and orientation measures and provide visual cues for radiologists to identify possible lesions more easily without increasing false positives.

In this paper, an artifact-free enhancement algorithm based on overcomplete multiscale representations is presented. First, an image was decomposed using a fast wavelet transform algorithm. At each level of analysis, energy and phase information are computed via a set of separable steerable filters. Then, a measure of coherence within each level was obtained by weighting an energy measure with the ratio of projections of local energy within a specified window. Each projection was computed onto the central point of a window with respect to the total energy within that window. Finally, a nonlinear operation, integrating coherence and orientation information, was applied to modify transform coefficients within distinct levels of analysis. These modified coefficients were then reconstructed, via an inverse fast wavelet transform, resulting in an improved visualization of significant mammographic features. The novelty of this algorithm lies in the detection of directional multiscale features and the removal of aliased perturbations. Compared to existing multiscale enhancement techniques, images processed with this method appeared more familiar to radiologists due to localized enhancement of features.
\end{abstract}

Index Terms - Coherence measure, multiscale analysis, oriented energy, overcomplete representations, separable steerable filters, wavelet basis.

\section{INTRODUCTION}

M AMMOGRAMS can detect tumors that are an eighth of an inch in diameter, while manual examination usually fails to detect tumors smaller than a half-inch. Screening for breast disease via mammography depends on observing local and distant changes in tissues. Important visual clues of breast cancer include preliminary signs of masses and calcification clusters [5], [21]. Unfortunately, at the early stages of breast cancer, these signs are very subtle and varied in appearance, making diagnosis difficult, challenging even for specialists [5], [10]. Radiographic signs of cancer are related to tumor mass and density, size, shape, smoothness of borders, and calcification distribution. Extraction of these

Manuscript received April 8, 1998; revised November 3, 1998.

C.-M. Chang is with the Department of Electrical and Computer Engineering, University of Florida, Gainesville, FL 32611 USA.

A. Laine is with the Department of Biomedical Engineering, Columbia University, New York, NY 10027 USA (e-mail: laine@bme.columbia.edu).

Publisher Item Identifier S 1089-7771(99)01794-X. features and enhancement of them can assist radiologists to locate suspicious areas more reliably [33].

Studies of multiscale representations based on wavelets have been carried out for mammographic feature analysis [17], [28], [30]. Laine et al. [17] used two overcomplete multiscale representations for contrast enhancement. Mammograms were reconstructed from transform coefficients modified at each level by nonlinear weighting functions. Qian et al. [28], [27] introduced tree-structured nonlinear filters for microcalcification cluster detection. An image was enhanced by tree-structured nonlinear filters with fixed parameters and adaptive order statistic filters. Richardson [30] applied linear and nonlinear filtering approaches to the analysis of mammograms. Here, a linear multiscale decomposition was obtained via a wavelet transform; a nonlinear multiscale decomposition employed a "mean curvature partial differential equation" filter and "weighted majority-minimum range" filter. In addition, $\mathrm{Li}$ et al. [18] extended a conventional multiresolution wavelet transform into a multiresolution and multiorientation wavelet transform. They applied directional wavelet analysis to capture orientation information within each mammogram.

Freeman and Adelson [6] first proposed the concept of steerable filters and applied it to several problems in the area of computer vision. With a set of "basis filters," one can adaptively steer a filter along any orientation. Hilbert transform pairs were constructed to find a local "oriented energy" measure and dominant orientation.

Later, Kass and Witkin [12] developed an algorithm for estimating the orientation of texture patterns. An orientation pattern was decomposed into a flow field, describing the direction of anisotropy, and a residual pattern was obtained by describing an image in a coordinate system built from the flow field. Texture orientation was estimated from Laplacian of Gaussian filters. More recently, Rao and Schunck [29] proposed a related algorithm based on the gradient of the Gaussian. Their algorithm incorporated a more sophisticated scheme for computing the coherence of the flow field.

In this paper, an enhancement algorithm based on overcomplete multiscale wavelet analysis is described. These redundant representations provide the property of shift invariance which is shown to be advantageous for applications of feature extraction and contrast enhancement. Features were extracted by separable steerable filters. A coherent image and phase information were then generated. A nonlinear function, integrating coherence and phase information, was then applied to transform coefficients at each level of analysis. Finally, an enhanced image was obtained via an inverse wavelet transform 
of these modified coefficients. The novelty and advantage of this algorithm compared to existing techniques lies in its detection of directional features and the lack of perturbations (artifacts) in reconstructed images. The latter is of great importance for applications in medical imaging.

\section{BACKGROUND}

In this section, we briefly describe the mathematical background and fundamental ideas used in subsequent sections.

\section{A. Wavelet Transforms}

Wavelet transforms have become acknowledged as a useful tool for many applications in signal and image processing. A function $\psi(x)$ is said to be a wavelet if and only if its Fourier transform $\hat{\psi}(\xi)$ satisfies the admissibility condition

$$
C_{\psi}=\int_{-\infty}^{+\infty} \frac{|\hat{\psi}(\xi)|^{2}}{|\xi|} d \xi<\infty
$$

This condition implies that

$$
\int_{-\infty}^{+\infty} \psi(x) d x=0
$$

which means that $\psi(x)$ will have at least some oscillations.

Wavelets constitute a family of functions derived from a single function $\psi$ (other wavelet) by dilations and translations

$$
\psi_{a, b}(x)=\frac{1}{\sqrt{a}} \psi\left(\frac{x-b}{a}\right)
$$

where $a \in R^{+}, b \in R$. A central idea in wavelet analysis is to represent any function $f(t)$ as a superposition of wavelets. The continuous wavelet transform is defined as

$$
W_{f}(a, b)=\left\langle f, \psi_{a, b}\right\rangle=\frac{1}{\sqrt{a}} \int_{-\infty}^{+\infty} f(x) \overline{\psi\left(\frac{x-b}{a}\right)} d x
$$

where $\bar{\psi}$ denotes the complex conjugate of $\psi$. A function can be reconstructed from its wavelet transform by means of the "resolution of identity" formula

$$
f=\frac{1}{C_{\psi}} \int_{-\infty}^{+\infty} \int_{-\infty}^{+\infty}\left\langle f, \psi_{a, b}\right\rangle \psi_{a, b} \frac{d a d b}{a^{2}} .
$$

In the application of digital mammography, one needs to express $f$ as a discrete superposition. If we discretize the translation and dilation parameters of the wavelet in (3), we see that

$$
\psi_{m, n}(x)=a_{0}^{-(m / 2)} \psi\left(a_{0}^{-m} x-n b_{0}\right)
$$

where $a=a_{0}^{m}, b=n b_{0} a_{0}^{m}$, with $m, n \in Z$, and $a_{0}>1, b_{0} \neq$ 0 . On this discrete grid, the wavelet transform is simply

$$
W_{f}(m, n)=a_{0}^{-(m / 2)} \int_{-\infty}^{+\infty} f(x) \psi\left(a_{0}^{-m} x-n b_{0}\right) d x .
$$

An original signal can be approximated as linear combinations of a wavelet basis

$$
f(x) \approx \sum_{m, n} W_{f}(m, n) \psi_{m, n}(x)
$$

One popular discretization is to choose $a_{0}=2, b_{0}=1$

$$
\psi_{m, n}(x)=2^{-(m / 2)} \psi\left(2^{-m} x-n\right)
$$

which are called dyadic wavelets.

\section{B. Steerable Filters}

A function $f(x, y)$ is called "steerable" if it can be expressed as a linear combination of rotated versions of itself. The fundamental idea of steerable filters is to apply distinct "basis filters" that correspond to a fixed set of orientations and interpolate between each discrete response. Thus, one must first decide the number of "basis filters" and corresponding interpolation functions. Let $\theta_{i}$ be the angle of some $i$ th basis filter and $k_{i}(\theta)$ denote an interpolation function. As defined in [6], a steering constraint may be formulated by

$$
f^{\theta}(x, y)=\sum_{i=1}^{L} k_{i}(\theta) f^{\theta_{i}}(x, y)
$$

where $L$ is the number of basis functions required to steer some function $f(x, y)$.

Hereafter, it will be more convenient to work in polar coordinates where $r=\sqrt{x^{2}+y^{2}}$ and $\phi=\arg (x, y)$. Let $f$ be any function that can be expressed as a Fourier series in polar angle $\phi$

$$
f(r, \phi)=\sum_{n=-N}^{N} a_{n}(r) e^{j n \phi}
$$

where $j=\sqrt{-1}$ and $N$ is the discrete length of the coefficients.

The theorems below were posed by [6] and are included for clarity and completeness of description.

Theorem 1: Steering condition (10) holds for a function $f$ expanded in the form of (11) if and only if the interpolations $k_{i}(\theta)$ are solutions of

$$
\left(\begin{array}{c}
1 \\
e^{j \theta} \\
\vdots \\
e^{j N \theta}
\end{array}\right)=\left(\begin{array}{cccc}
1 & 1 & \cdots & 1 \\
e^{j \theta_{1}} & e^{j \theta_{2}} & \cdots & e^{j \theta_{L}} \\
\vdots & \vdots & \ddots & \vdots \\
e^{j N \theta_{1}} & e^{j N \theta_{2}} & \cdots & e^{j N \theta_{L}}
\end{array}\right)\left(\begin{array}{c}
k_{1}(\theta) \\
k_{2}(\theta) \\
\vdots \\
k_{L}(\theta)
\end{array}\right) .
$$

Thus, $f^{\theta}(r, \phi)$ may be expressed as

$$
f^{\theta}(r, \phi)=\sum_{i=1}^{L} k_{i}(\theta) g_{i}(r, \phi)
$$

where $g_{i}(r, \phi)$ can be any set of functions.

Theorem 2: Let $f(x, y)=W(r) P_{N}(x, y)$, where $W(r)$ is an arbitrary windowing function, and $P_{N}(x, y)$ is an $N$ th order polynomial in $x$ and $y$, whose coefficients may depend on $r$. Linear combinations of $2 N+1$ basis functions are sufficient to synthesize $f(x, y)=W(r) P_{N}(x, y)$ rotated to any angle. If $P_{N}(x, y)$ contains only even (or odd) order terms, then $N+1$ basis functions are sufficient. 

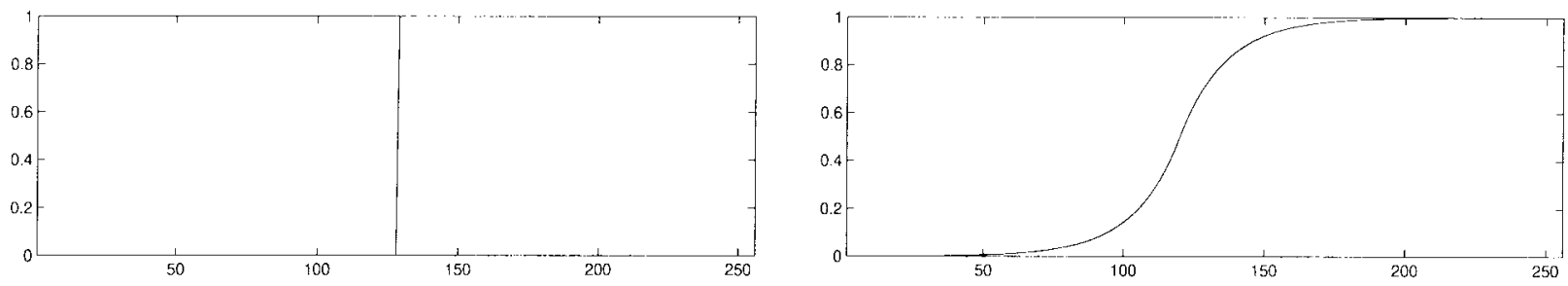

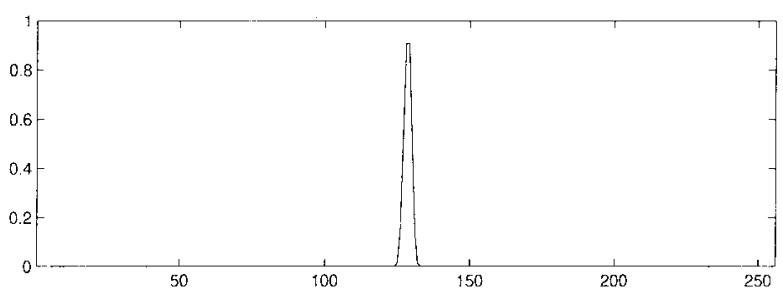

(a)
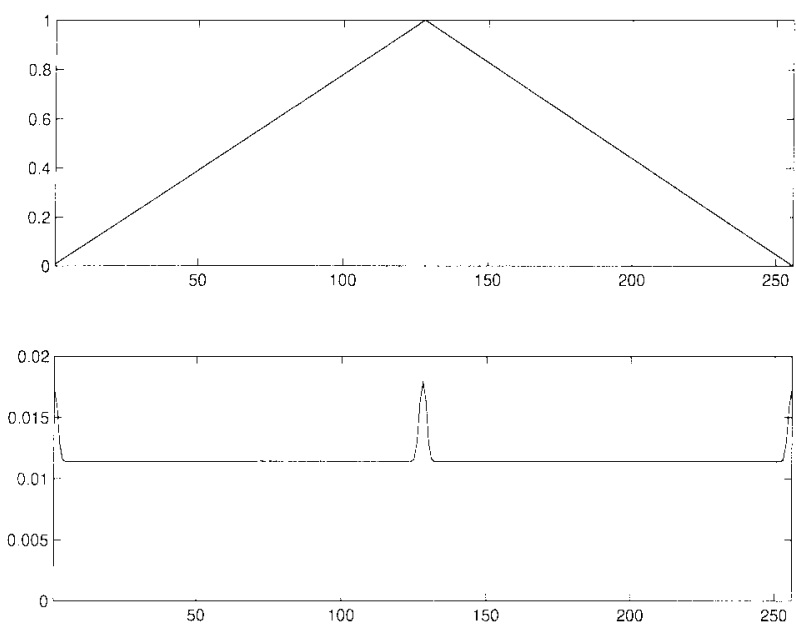

(c)

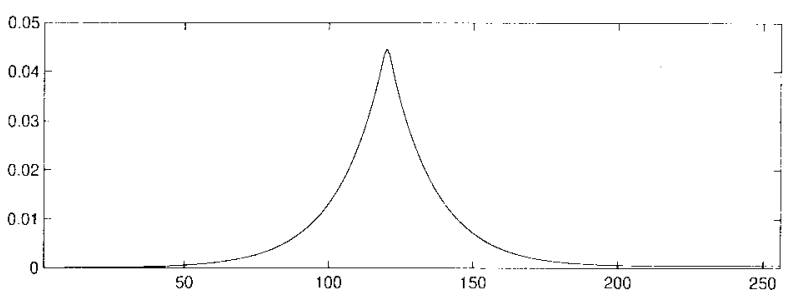

(b)
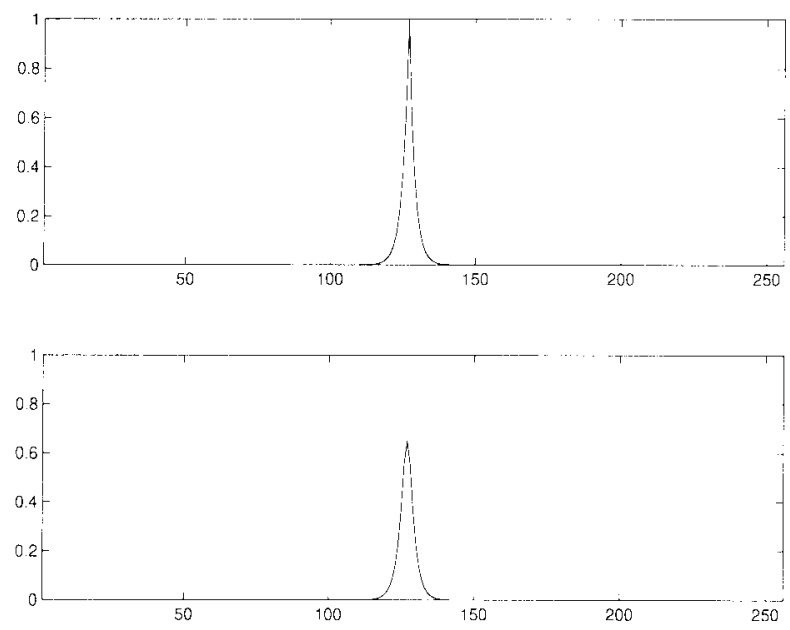

(d)

Fig. 1. Energy functions of four signal models. (a) Ideal step edge. (b) Smoothed step edge. (c) Roof edge. (d) Line (spike edge).

\section{Local Energy}

Relying on physiological evidence, a local energy operator was first proposed by Morrone and Owens [22]. Rather than considering differential properties, they studied the properties of the Fourier expansion of an intensity function. They demonstrated that maxima of an energy function were coincident with the points of maximum phase congruency. A local energy model has an advantage in that it simultaneously detects both edges and lines. Some one-dimensional (1-D) examples are shown in Fig. 1. The top rows show the original signals and the bottom rows show the norms of local energy functions. We observe that the local maxima of each energy function occurs at the central position of each feature in the signal model.

Let $I G(\theta)$ and $I G^{H}(\theta)$ be the outputs of constituent filter $G(\theta)$ and its quadrature pair $G^{H}(\theta)$, respectively. An oriented energy function can be expressed by the squared outputs of a quadrature pair of filters steered to an angle $\theta$, that is,

$$
\begin{aligned}
E(\theta) & =[I G(\theta)]^{2}+\left[I G^{H}(\theta)\right]^{2} \\
& =[G(\theta) * I]^{2}+\left[G^{H}(\theta) * I\right]^{2}
\end{aligned}
$$

where $I$ denotes an image.
Since convolution is a linear operation, from (10), (14) can be reformulated as

$$
E(\theta)=\left[\sum_{i=1}^{L_{1}} k_{i}(\theta) g_{i}\right]^{2}+\left[\sum_{j=1}^{L_{2}} k_{j}^{H}(\theta) g_{j}^{H}\right]^{2}
$$

where $L_{1}$ and $L_{2}$ are the numbers of functions required to steer functions $G(\theta), G^{H}(\theta), k_{i}(\theta)$, and $k_{j}^{H}(\theta)$ denote interpolation functions, and

$$
\begin{gathered}
g_{i}=G_{i} * I \\
g_{j}^{H}=G_{j}^{H} * I
\end{gathered}
$$

are the outputs of each basis filter and its quadrature pair, respectively, which are known constants. Equation (15) is a function of both $k_{i}(\theta)$ and $k_{j}^{H}(\theta)$ that are sinusoidal functions of $\theta$ [6], [3] and therefore a function of $\theta$.

Equation (15) contains squares of sinusoidal functions. Following [6], this expression can be simplified to a Fourier series in angular form via substitutions obtained by the trigonometric identity (recall, for example, $\sin ^{2} \theta=(1-\cos (2 \theta)) / 2$ ) and 


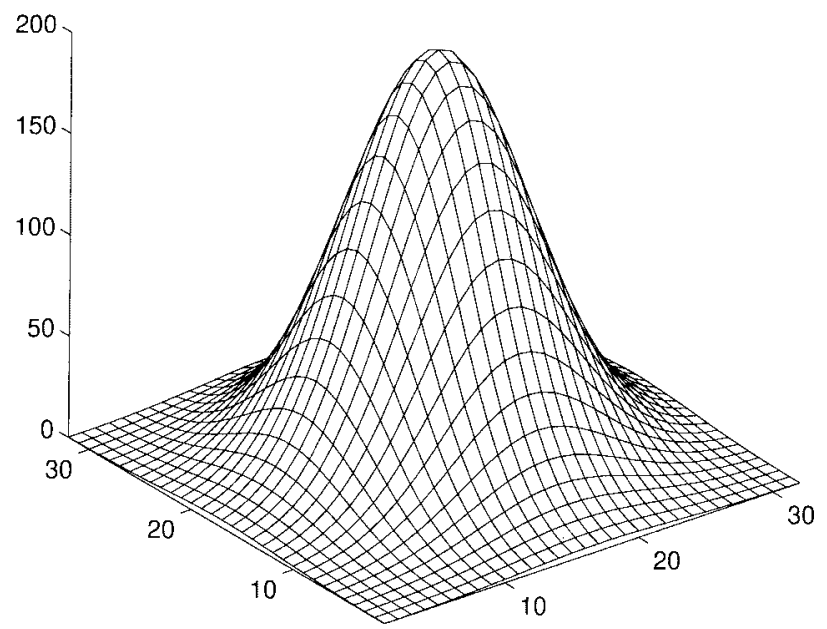

(a)

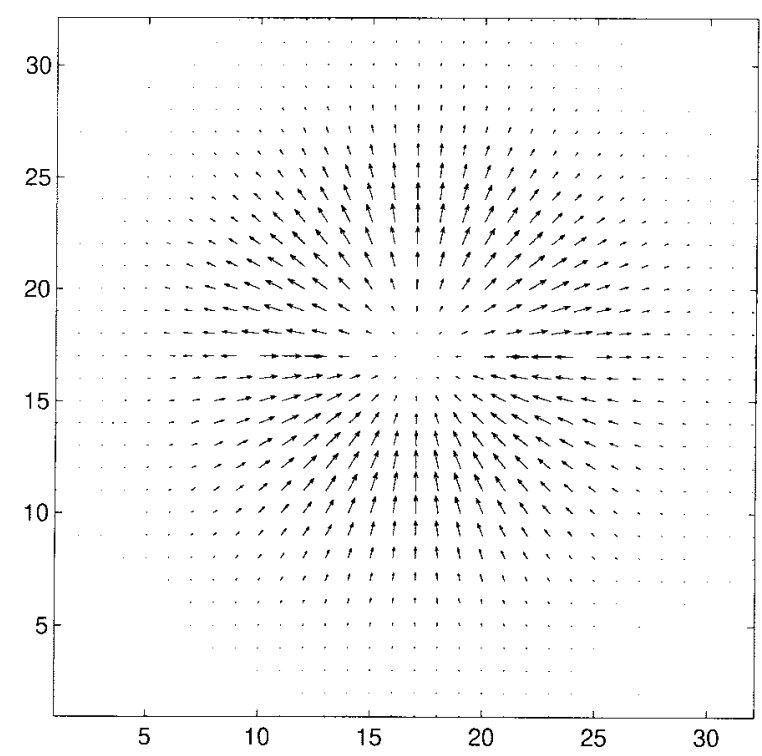

(b)

Fig. 2. An example of oriented energy map construction. (a) A test function. (b) Orientation and magnitude map of a test function.

$$
\begin{aligned}
& \left.\left.\cos ^{2} \theta=(1+\cos (2 \theta)) / 2\right)\right) \\
& \begin{aligned}
E(\theta)= & a_{0}+a_{1} \cos (2 \theta)+a_{2} \sin (2 \theta) \\
& +[\text { higher order terms } \cdots] .
\end{aligned}
\end{aligned}
$$

The lower frequency terms of this identity were used to approximate the dominant directions $A$ and their associated magnitudes $M$

$$
\begin{aligned}
A & =\arctan \left[a_{1}, a_{2}\right] / 2, \\
M & =\sqrt{a_{1}^{2}+a_{2}^{2}}
\end{aligned}
$$

where the $\arctan \left[a_{1}, a_{2}\right]$ function calculates the arc tangent of $\left(a_{1} / a_{2}\right)$ and the signs of both arguments $a_{1}$ and $a_{2}$ are used to determine the quadrant of the associated result.

Fig. 2 shows the oriented energy map of a two-dimensional (2-D) Gaussian function

$$
f(x, y)=e^{-\left(x^{2}+y^{2}\right)} .
$$

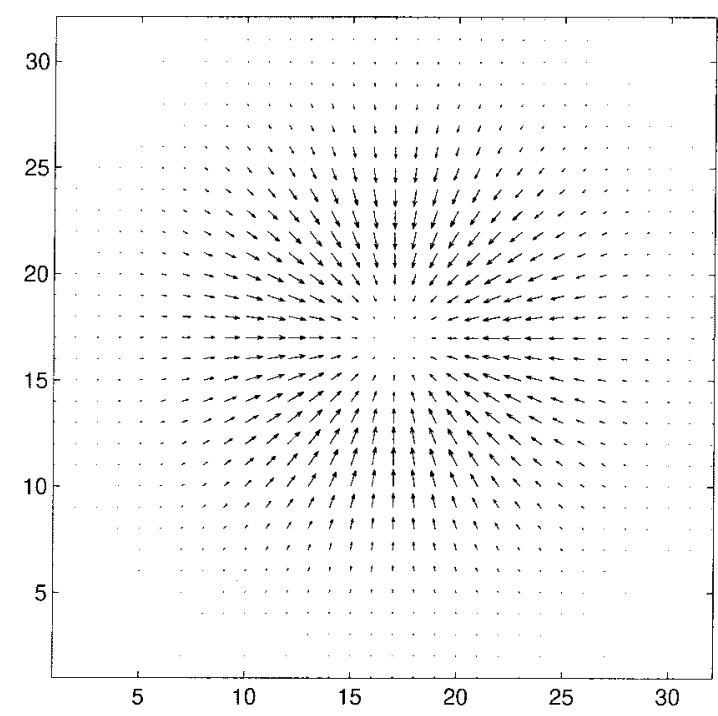

Fig. 3. Corrected oriented energy map.

A test function is shown in Fig. 2(a). Using a second derivative of a Gaussian function and its Hilbert transform to measure oriented energy, we plotted the direction and magnitude of each position on a map where the arrow indicates direction and length denotes magnitude, as shown in Fig. 2(b). Edges were detected at inflection points of the surface. The magnitudes of the energy function are maxima, therefore the lengths are longer than other points of the function.

Note that Fig. 2(b) does not show the correct phase in the upper half of the map. All arrows are pointing outward instead of inward. There is a $180^{\circ}$ phase shift since the oriented energy is obtained from the squared outputs of quadrature pairs. We applied a simple algorithm to correct this phase shift. First, we divide the space into eight directions and approximate the phase at each point to exactly one of these discrete orientations. Next, the neighbors of each point along each approximated direction are examined. If the intensities along a direction are increasing, the phase is not changed; otherwise, we correct the phase by a $180^{\circ}$ shift. A sample corrected oriented energy map is shown in Fig. 3.

\section{Measure of Coherence}

Texture plays an important role in many machine vision and image processing tasks including surface inspection, object classification, surface orientation, and shape determination [2]. Texture patterns may be characterized by extracting measurements that quantify both the "nature" and directions of patterns. Most breast carcinomas have the appearance of stellate lesions consisting of a central mass surrounded by radiating spicules [5]. The spicules may radiate outward in any direction and vary in length. These features provide important clues for the early detection of such lesions.

Much attention has been given to the notion of decomposing an intensity image into intrinsic images to extract meaningful information [1], [20]. Such intrinsic properties represent basic components of some image formation process and therefore can reveal features "hidden" inside an image. The information provided is not obvious from the intensity image alone. 


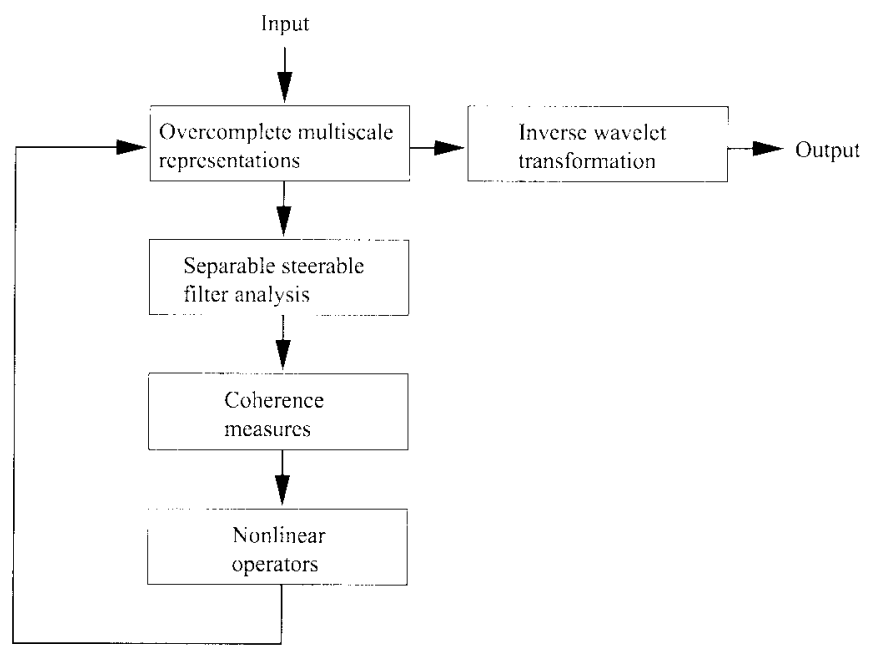

Fig. 4. Block diagram of the proposed algorithm.

Rao and Schunck [29] defined an orientation field of a texture image to consist of two "images" - an angle image and a coherence image. The angle image denotes the dominant local orientation at each point while the coherence image represents the degree of anisotropy at each point. The application of angle and coherence images as intrinsic images was strongly advocated. In this paper, we investigated the efficiency of these two representations to capture and enhance features of importance to mammography screening.

\section{Methodology}

Our algorithm consists of four steps [3], [4]: 1) construction of an overcomplete multiscale representation; 2) analysis via separable steerable filters; 3 ) computing coherence measures; and 4) application of nonlinear operators, as shown in Fig. 4. Next, we describe each step in the sections below.

1) Overcomplete Multiscale Representations: Through multiscale analysis, one can extract features at distinct scales and "mine" subtle information often hidden in an original mammogram [17]. One major drawback of wavelet transforms is their lack of translation invariance [32], making the content of wavelet subbands unstable under the translations of an input signal. In our algorithm, a digitized mammogram was decomposed using a fast wavelet transform algorithm (FWT) [19]. In order to obtain wavelet coefficients at each level without downsampling, an undecimated "algorithme à trous" (algorithm with holes) [9], [31] was implemented. In the spatial domain, these redundant representations correspond to signals without aliasing.

Fig. 5 shows the differences between traditional wavelet representations and overcomplete multiscale representations. Here, we demonstrate two levels of a decomposition. An original image is shown on the left-hand side of Fig. 5(a). A sample region $(4 \times 4)$ is denoted by dots in Fig. 5(a). After decomposition, corresponding wavelet representations are shown on the right-hand side of Fig. 5(a) (the upper left corner shows the approximated image). Distinct pixels, at each level of analysis, corresponding to the sample region are also shown. Because of downsampling at each level, there are four pixels corresponding to the original sample region (16 pixels) at level one; while at level two, there is only a single pixel. Thus, if we change a value of one pixel at level two, there will be 16 pixels affected in the original image matrix. Fig. 5(b) shows an overcomplete multiscale representation where the approximated image is shown on the right-hand side of the level 2 decomposition. At each level, there is exactly one pixel corresponding to each pixel in the original image.

Let $S^{0}$ denote an original image and $D^{i} h$ be obtained by inserting $2^{i}-1$ zeros between every pair of coefficients representing $h .\left(D^{i} h\right)_{x}$ and $\left(D^{i} h\right)_{y}$ stand for carrying out convolution operations with filter $D^{i} h$ along the $x$ and $y$ directions, respectively. Then, the decomposition and reconstruction equations for level $i$ are computed as follows.

Decomposition:

$$
\begin{aligned}
S^{i+1} & =S^{i} *\left(D^{i} h\right)_{x} *\left(D^{i} h\right)_{y} \\
W_{x}^{i+1} & =S^{i} *\left(D^{i} g\right)_{x} \\
W_{y}^{i+1} & =S^{i} *\left(D^{i} g\right)_{y} .
\end{aligned}
$$

\section{Reconstruction:}

$$
\begin{aligned}
S^{i}= & W_{x}^{i+1} *\left(D^{i} k\right)_{x} *\left(D^{i} l\right)_{y}+W_{y}^{i+1} *\left(D^{i} l\right)_{x} \\
& *\left(D^{i} k\right)_{y}+S^{i+1} *\left(D^{i} \bar{h}\right)_{x} *\left(D^{i} \bar{h}\right)_{y}
\end{aligned}
$$

where " $*$ " indicates discrete circular convolution, and $h, g, k$, and $l$ are filters whose Fourier transforms $(H(\omega), G(\omega), K(\omega)$, and $L(\omega)$, respectively) satisfy [19]

$$
\begin{aligned}
G(\omega) K(\omega)+|H(\omega)|^{2} & =1 \\
L(\omega) & =\frac{1+|H(\omega)|^{2}}{2} .
\end{aligned}
$$

Following [19], we choose $G(\omega)$ to approximate the derivative of a smoothing function, which is equivalent to a high-pass filter; $H(\omega)$ is a smoothing function, which is equivalent to a low-pass filter. The block diagrams for decomposition and reconstruction operations at level $i$ are shown in Fig. 6(a) and (b), respectively.

2) Analysis via Separable Steerable Filters: A filter is called "steerable" if the filter at an arbitrary orientation can be expressed as a linear combination of a set of basis filters, generated from rotations of a single kernel [6]. Steerable filters [6], [14], [13], which can be adaptively adjusted to arbitrary orientation, were used to detect stellate patterns of spicules and locate feature orientations more precisely. As pointed out in [26], the separability property of discrete filters can speed up computation considerably when convolved with a large image matrix. In our algorithm, we used three basis functions as steerable filters. The $x-y$ separable steerable approximations of filter kernels were generated by a singular value decomposition (SVD) algorithm [6], [26]. Using a set of separable steerable filters, the magnitude $\left(M^{i}\right)$ and associated dominant directions $\left(A^{i}\right)$ of local energy were determined by the basis functions and their Hilbert transforms [6], [11], [22], [24]. Fig. 7 illustrates the procedure of steerable filter processing.

We applied separable steerable filters to an original image $S^{0}$ and a low-pass filtered image at each level $S^{i}, i=1, \cdots n$, to distill salient multiscale features. The three basis functions 


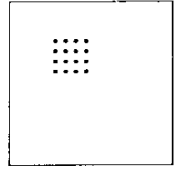

Original image

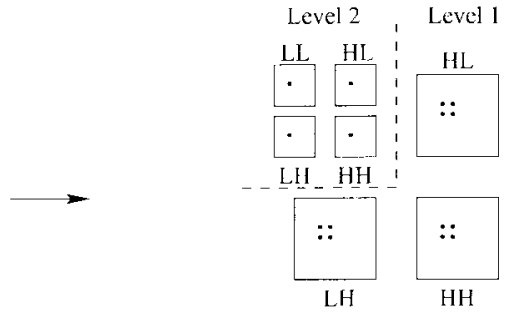

Wavclet representations

(a)

Level 1

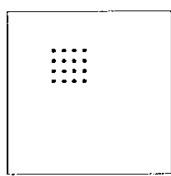

Original image

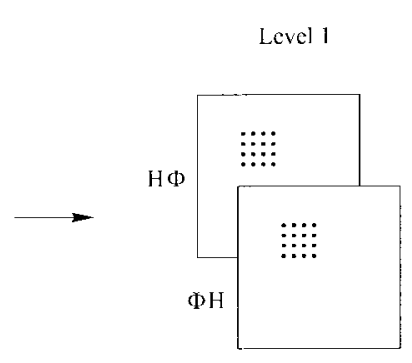

Wavelet representations
Level 2

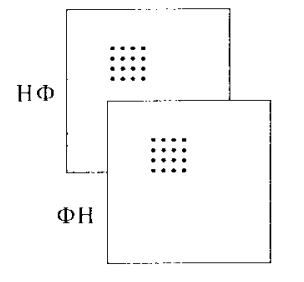

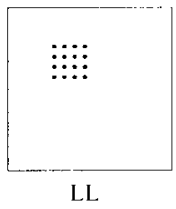

LL

(b)

Fig. 5. Illustrations of distinct wavelet representations: (a) Traditional wavelet representations. (b) Overcomplete multiscale representations. (Note that the first character denotes an operation along the $x$ axis, and the second, the $y$ axis. L: low-pass filtering. H: high-pass filtering. $\Phi$ remains unchanged.)

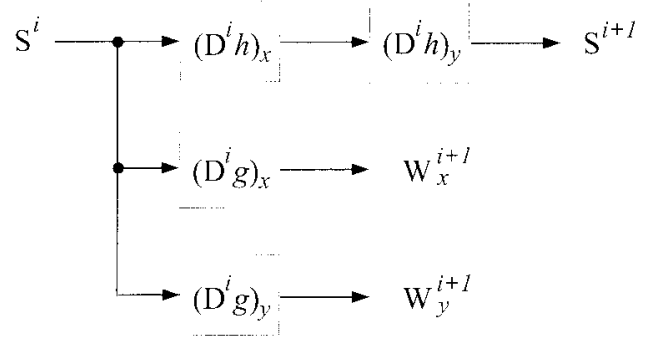

(a)

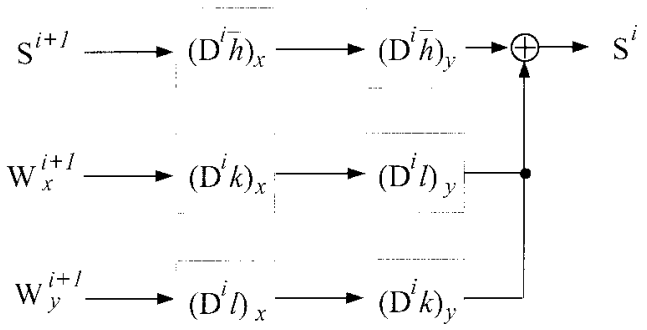

(b)

Fig. 6. Block diagram of fast wavelet transform at level i. (a) Decomposition, (b) Reconstruction.

which were applied in our implementation algorithm were discretized and are shown in Fig. 9. The Hilbert transform of the basis function was thereafter obtained by [7].

To illustrate how dominant directions of local energy relate to image features, a sample mammogram which was extracted from Fig. 17(a), as shown in Fig. 8. Fig. 8(a) presents a region of interest (ROI) image matrix of $32 \times 32$. The corresponding gradient is displayed in Fig. 8(b). After applying steerable filters and an energy function to locate features, the resulting magnitude and orientation map is shown in Fig. 8(c). Compar-

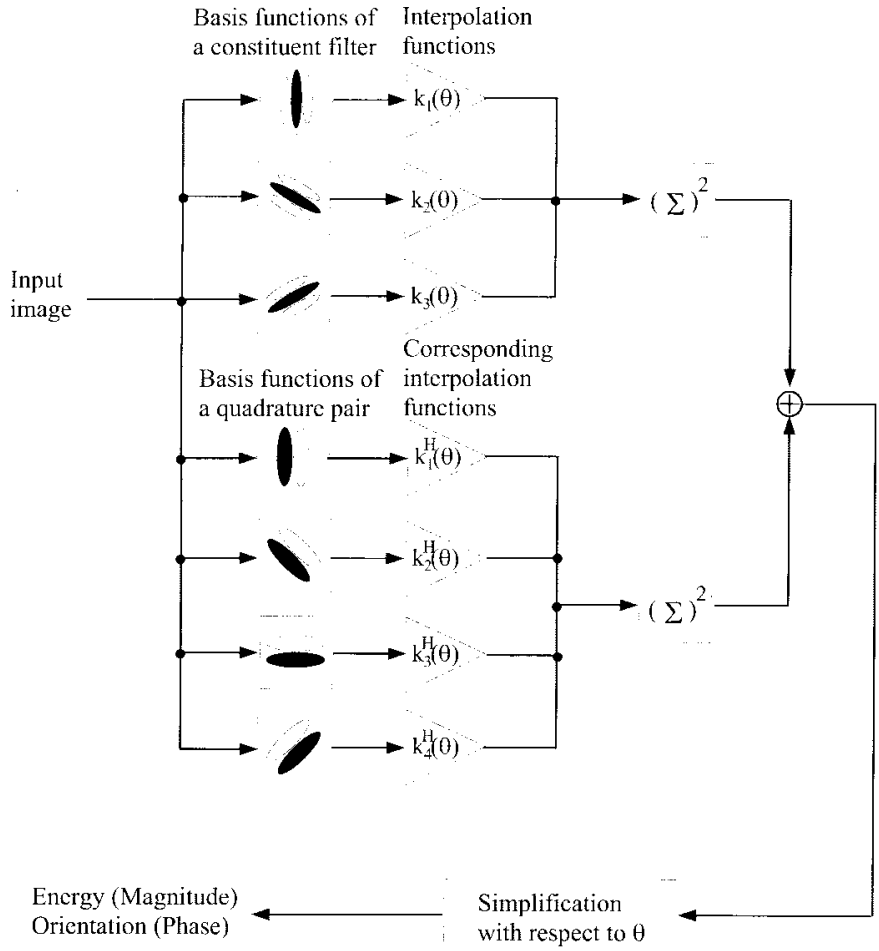

Fig. 7. Diagram of steerable filter processing.

ing Fig. 8(b) and (c), note that the map obtained from steerable filters detected the boundary more precisely and with less variation.

3) Coherence Measures: A coherence map is an image showing a local measure of the degree of anisotropy of flow [12], [29]. If orientations of a texture pattern at any point $\left(x_{i}, y_{i}\right)$ are coherent, then magnitude and phase information 


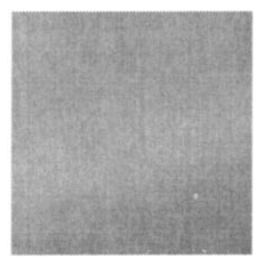

(a)

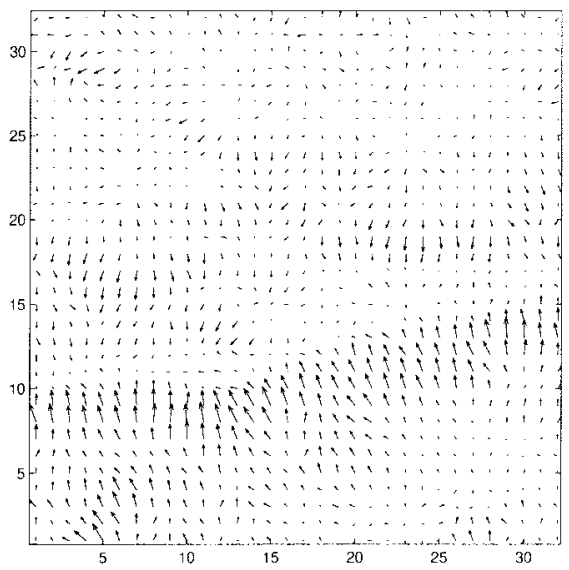

(b)

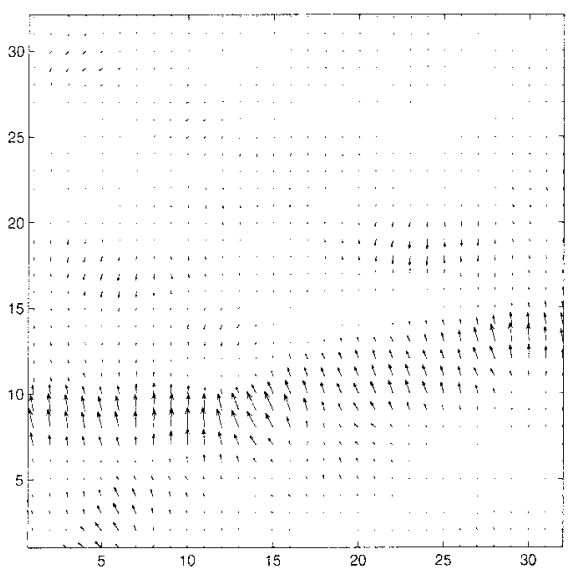

(c)

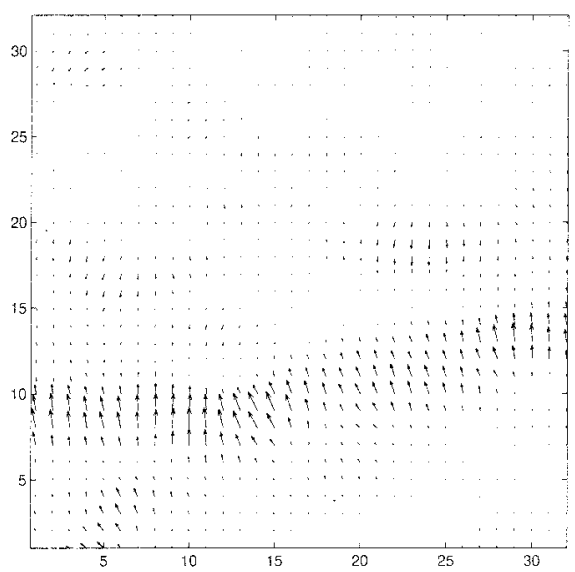

(d)

Fig. 8. A mammogram sample ROI showing computed features derived from steerable filters and an energy function. (a) An extracted sample image. (b) The gradient map. (c) Local energy. (d) Coherence map.

are important and should be emphasized. Conversely, if the orientations are not coherent, the magnitude and phase infor-

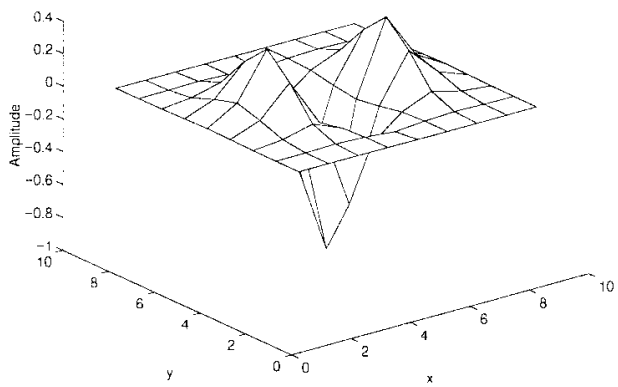

(a)

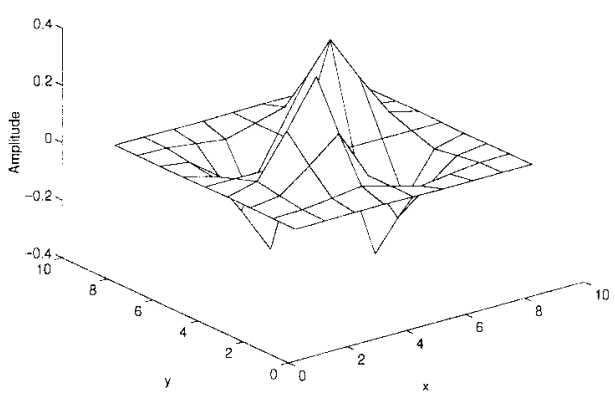

(b)

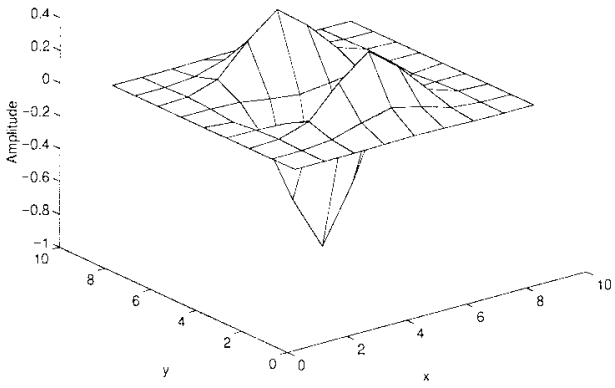

(c)

Fig. 9. Two-dimensional impulse responses of three steerable basis functions. (a)-(c) Input to $k_{1}(\theta), k_{2}(\theta)$, and $k_{3}(\theta)$, respectively, as shown in Fig. 7.

mation can be neglected or attenuated. Kass and Witkin [12] suggested a simple way for measuring strength of coherence by finding the ratio

$$
\chi(j, k)=\frac{|W(j, k) J(j, k)|}{(W(j, k)|J(j, k)|)}
$$

where $W(j, k)$ denotes a local weighting function with unit integral, $J(j, k)$ denotes the squared gradient vector at $(j, k)$, and $|\chi|$ denotes the absolute value of $\chi$.

An alternative measure of coherence was proposed by Rao and Schunck [29] and was obtained by weighting energy with a normalized projection of the energy within a specified window $(\mathcal{W})$ onto the central point $(j, k)$ of each window. The coherence measure $\left(C^{i}\right)$ was expressed as

$$
\begin{aligned}
& C^{i}(j, k)=M^{i}(j, k) \\
& \frac{\sum_{(m, n) \in \mathcal{W}}\left|M^{i}(m, n) \cos \left(A^{i}(j, k)-A^{i}(m, n)\right)\right|}{\sum_{(m, n) \in \mathcal{W}} M^{i}(m, n)}
\end{aligned}
$$




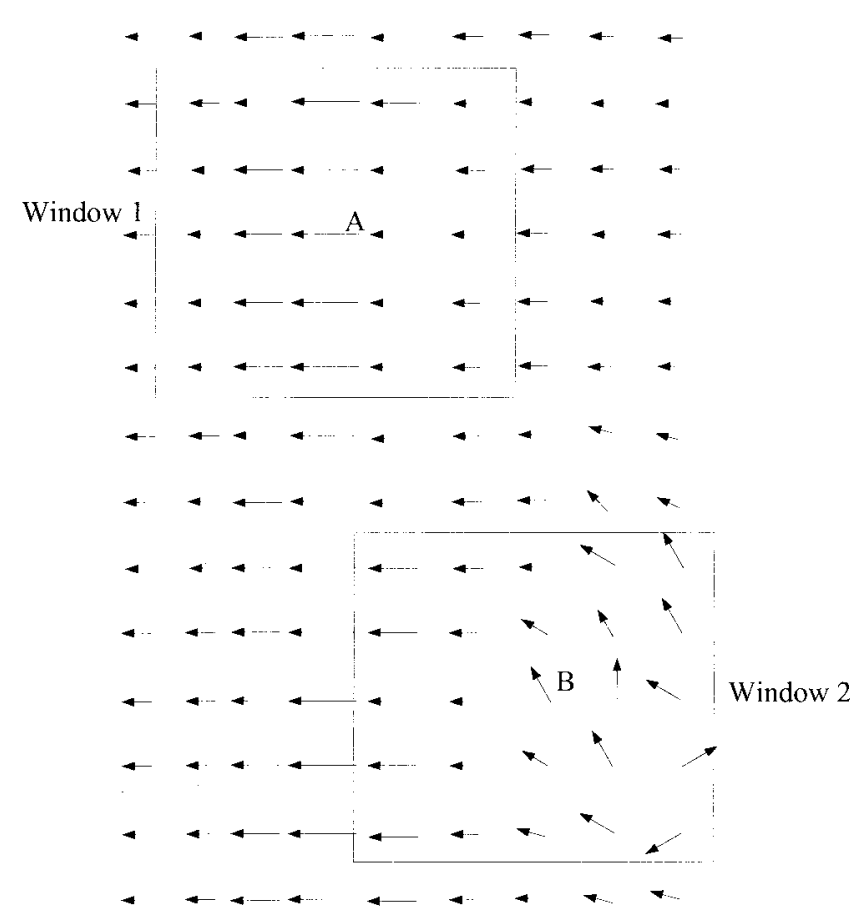

Fig. 10. An example showing the effectiveness of a coherence measure.

where $M^{i}(j, k)$ and $A^{i}(j, k)$ denote energy and phase of point $(j, k)$ at level $i$, respectively. This coherence measure incorporates the gradient magnitude and hence places more weight on regions that have higher visual contrast.

Let us examine more closely how a coherence measure works. Suppose we choose a region somewhere in an image, as shown in Fig. 10. Pixel $A$ is on a vertical edge line because its magnitude is maximum along the direction of its phase, i.e., there is an edge along the vertical direction of pixel $A$. The coherence at pixel $A$ is measured within window 1 which surrounds pixel $A$. Since the pixels inside window 1 have the same phase as pixel $A$, the coherence of pixel $A$ is equal to the magnitude at this pixel. While the pixels, surrounding pixel $B$, inside window 2 point to distinct directions, the projections of these pixels on pixel $B$ will be "stable." The coherence at pixel $B$ is thus reduced. This pixel can be considered a "disturbance."

Experimentally, this method outperformed previous measures applied to similar data [29]. In our algorithm, we implemented this approach and measured each coherence map. In order to capture multiscale features, we changed the window size at each distinct level of analysis. At the finest level, a $5 \times 5$ window was used, while for the coarser levels larger windows (e.g., $7 \times 7$ or $9 \times 9$ ) were used. For simplificity of implementation, an odd window size was selected at each level. Fig. 11 shows an example of a $5 \times 5$ window used to carry out this operation. The actual measure of coherence $C^{i}$ was obtained from (22).

Fig. 8(d) shows the coherence map computed from Fig. 8(c). Note that this map retained the magnitudes of edge pixels (the pixels which are local maxima in each corresponding orientation) while attenuating surrounding pixels. The net result is that edges become sharper. For those pixels of which surrounding pixels had "disturbances," their

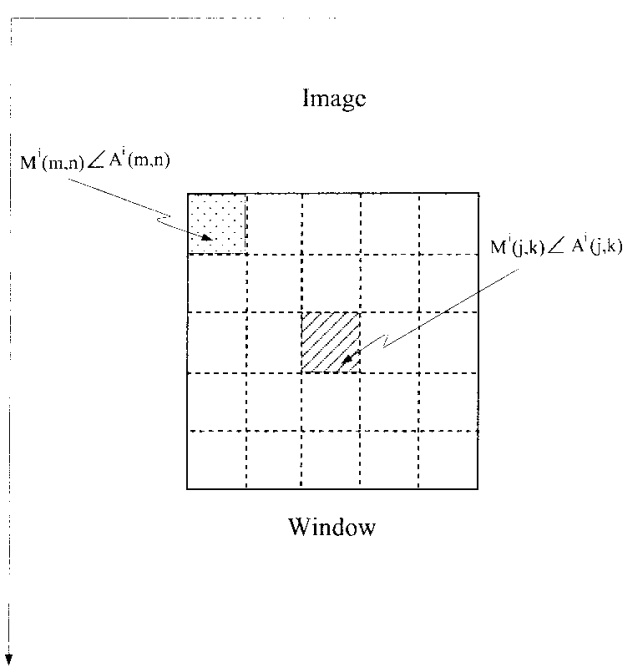

Fig. 11. Illustration of measuring the coherence of an image.

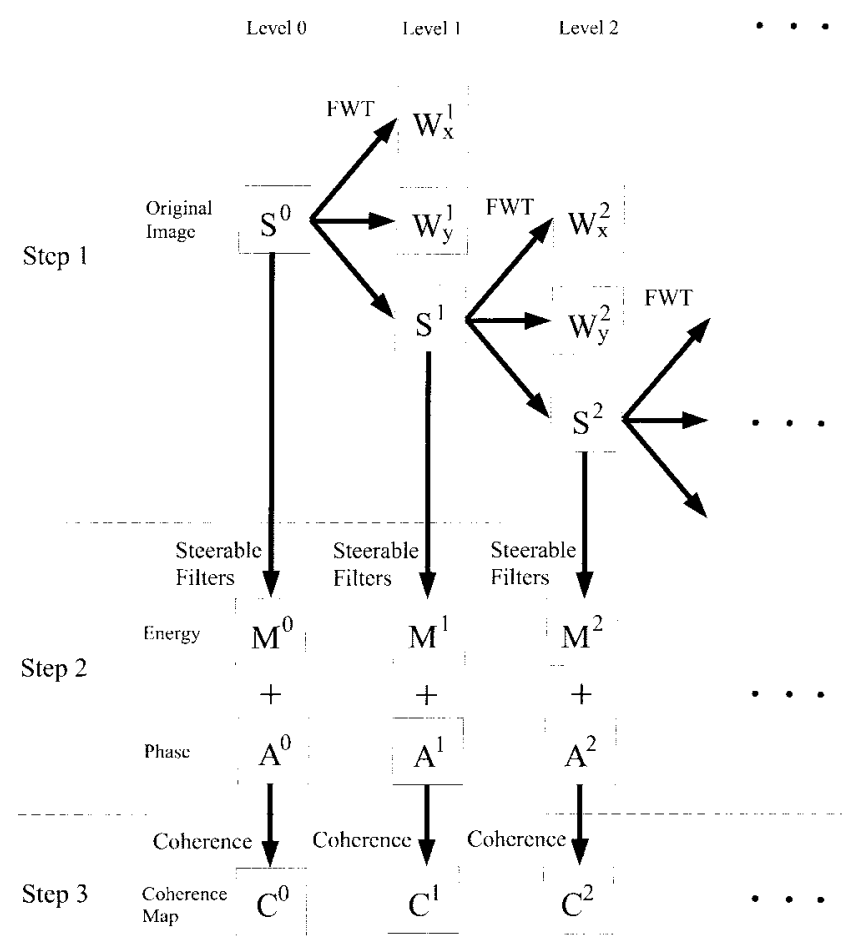

Fig. 12. Overview of processing for Steps 1-3.

magnitudes were attenuated and were not considered features. The combination of coherence and orientation structure was able to extract the more salient features of spiculated lesions. For an overview, a schematic diagram of processing Steps $1-3$ is shown in Fig. 12.

4) Nonlinear Operators: We have now computed all the information needed to complete the algorithm. A nonlinear operation is next applied within each level to precisely modify transform coefficients. This final operation integrates both coherence map and phase information, as described below.

a) Modification from coherence maps: Let $C^{i}(j, k)$ denote the coherence measure of point $(j, k)$ at some level $i$. First, we find the local maxima of the coherence map within each level. These maxima correspond to features in an image 
and their propagations at distinct levels of analysis. Therefore, they are likely candidate locations to "boost" or amplify. We call these maps $\left(C_{f}^{i}\right)$ feature maps. Next, the maxima or square roots of these maxima are mapped onto a 256-value intensity scale, depending on the threshold value of the scale $\left(S_{t}^{i}\right)$ at the first level, that is,

$$
\begin{cases}255 \frac{C_{f}^{i} C_{f, \text { min }}^{i}}{C_{f, \text { max }}^{i}-C_{f, \text { min }}^{i}}, & \text { if } S_{t}^{1}<S_{T} \\ 255 \frac{\sqrt{C_{f}^{i}}-\sqrt{C_{f, \text { min }}^{i}}}{\sqrt{C_{f, \text { max }}^{i}}-\sqrt{C_{f, \text { min }}^{i}}}, & \text { otherwise }\end{cases}
$$

where $C_{f, \max }^{i}$ and $C_{f, \min }^{i}$ are the maximum and minimum of $C_{f}^{i}$, respectively. $S_{T}$ is a selected threshold value. We compute the square root of the maxima because the difference between the maximal values may be large (especially for the first two levels of analysis). This operation prevents most values from mapping onto a small scale.

We then construct a histogram of square roots of local maxima and accumulate the number of mappings, from 1 up to some scale $\left(S_{t}^{i}\right) . S_{t}^{i}$ is defined as the "threshold scale" such that the accumulated number of local maxima from the histogram is over $99 \%$ of the total local maxima. The corresponding value of that scale is then chosen as the threshold value $\left(C_{f, t}^{i}\right)$ for that level of analysis.

Modifications of coherence maps were obtained by a nonlinear function expressed by

$$
C_{\text {mod }}^{i}(j, k)= \begin{cases}\sqrt{\frac{C^{i}(j, k)}{C_{\text {mean }}^{i}(j, k)}}, & \text { if } C^{i}(j, k)<C_{f, t}^{i}(j, k) \\ \sqrt{\frac{C_{f, t}^{i}(j, k)}{C_{\text {mean }}^{i}(j, k)}}, & \text { otherwise }\end{cases}
$$

where $C_{\text {mean }}^{i}(j, k)$ is the mean value of the figure map at each $i$ th level. For an image with a black background, the background pixels are not counted in computing the mean value. The square root values were computed so that large local maxima would not be over-emphasized and diminish important, more subtle local maxima.

b) Modification from phase: Phase information is important to distinctly characterize oriented texture. Therefore, we did not neglect its contribution in the modification of multiscale coefficients. We applied a sinusoidal weighting to phase information. The detailed subbands of wavelet coefficients obtained in Step 1, as shown in Fig. 1, include two components: a component along the $x$ direction and a component along the $y$ direction. The $x$ component was obtained by high-pass filtering along the $x$ direction, hence detecting mostly vertical features within the mammogram. We emphasized the points whose dominant orientations were near 0 and $\pi$ (with respect to the vertical axis). Thus, the modification from phase information was computed by

$$
A_{\text {mod }}^{i}(j, k)=\left|\cos \left(A^{i}(j, k)\right)\right| \text {. }
$$

The $y$ component was obtained by high-pass filtering along the $y$ direction, detecting mostly horizontal features. We emphasized the points whose dominant orientations were near $\pi / 2$
Level i

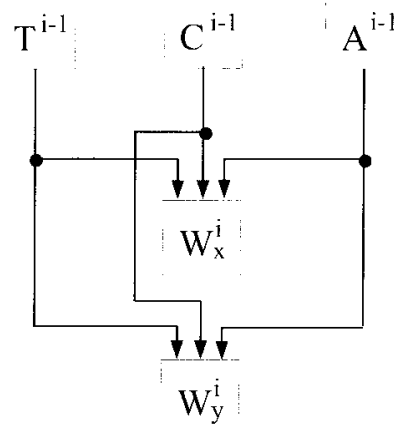

Fig. 13. A schematic diagram of nonlinear operator at level $i$.

and $3 \pi / 2$ (with respect to the vertical axis). Transform coefficients within each level were modified by phase information by

$$
A_{\text {mod }}^{i}(j, k)=\left|\sin \left(A^{i}(j, k)\right)\right| .
$$

Modifications from the coherence map and phase at level $i-1$ were combined to adjust each wavelet coefficient within level $i$. The resultant modification formula is therefore

$$
W_{\mathrm{mod}}^{i}=T^{i-1} \cdot C_{\mathrm{mod}}^{i-1} \cdot A_{\mathrm{mod}}^{i-1} \cdot W^{i}
$$

where $T^{i}$ was a constant at each level, adjusted as follows. Since the coefficients at finer levels of analysis may be contaminated by noise, the corresponding coherence maps may be corrupted. In addition, since the coherence map at the coarsest level is smooth, the adjustment at this level is more stable. In our study, we set the $T^{J}(J=$ coarsest level) as

$$
\sqrt{\frac{C_{f, \max }^{J}}{C_{f, t}^{J}}}
$$

Other levels were computed by

$$
T^{i}=\frac{C_{f, t}^{J}}{C_{f, t}^{i}} T^{J}
$$

where $i=0, \cdots, J-1$.

A schematic diagram of this nonlinear operator at level $i$ is shown in Fig. 13. Finally, the modified coefficients were used to reconstruct via an inverse fast wavelet transform. This resulted in an enhanced visualization of mammographic features, as shown in the next section.

\section{EXPERIMENTAL RESULTS AND DISCUSSION}

In this section, we present sample results of processed images. First, we show a 1-D example which does not take advantage of oriented information. We perform an overcomplete wavelet transform to the signal and decompose it into five levels of representation. The enhanced signal and the original signal are plotted adjacent in Fig. 14 for comparison. The transition regions in the original signal are clearly enhanced.

Our algorithm was designed with the goal of enhancing mammograms via oriented information. In order to capture distinct directions of subtle features, steerable filters were used. 


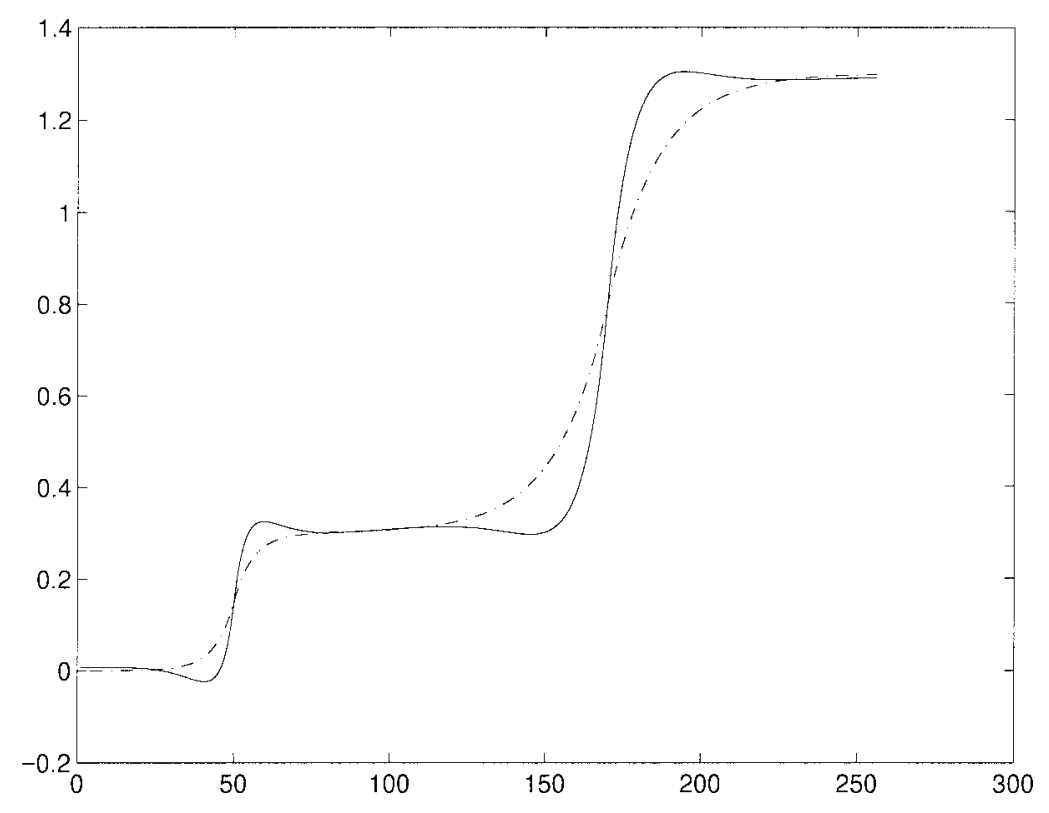

Fig. 14. Enhanced signal profile (solid line) overlaid with the original signal (dashed-dotted line).

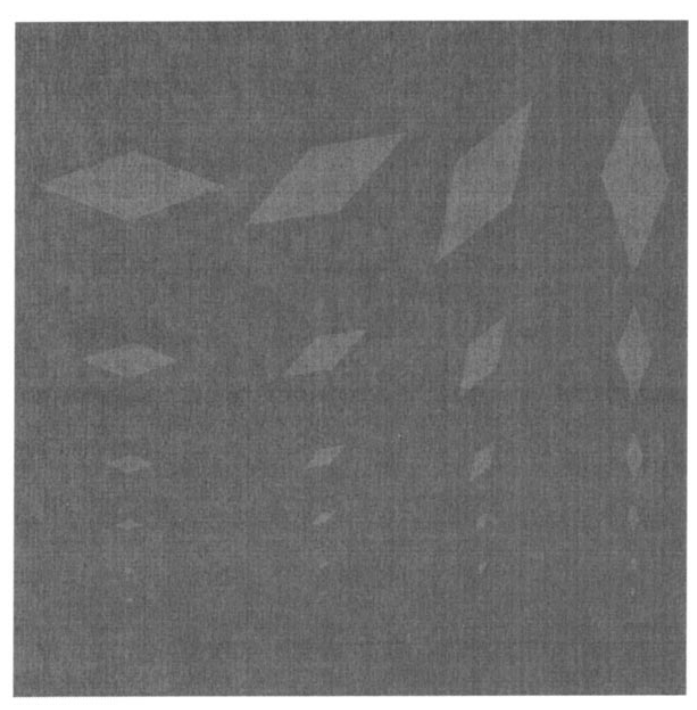

(a)

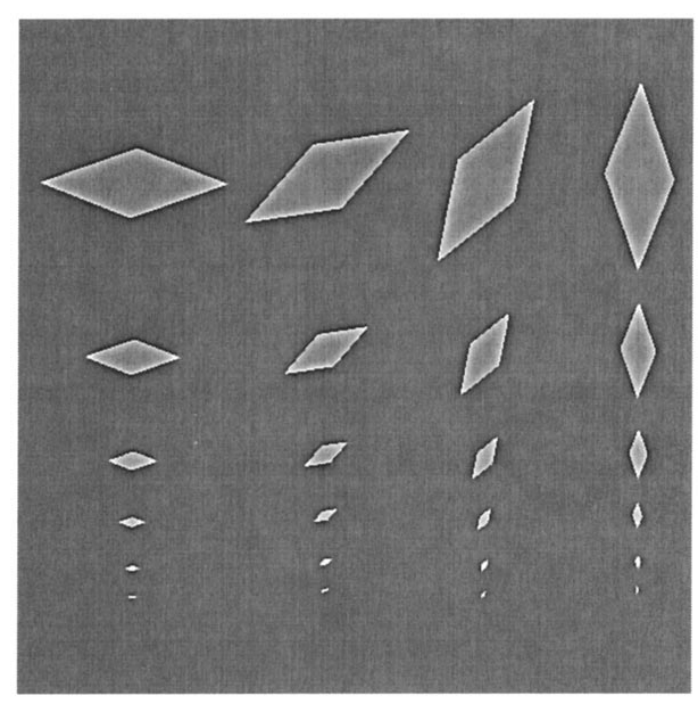

(b)

Fig. 15. The ability of detecting objects oriented along distinct directions at various scales: (a) Original test image (mathematical phantom). (b) Enhanced image after processing.

Fig. 15 shows the capability of steerable filters for detecting and enhancing objects of distinct direction and scale. The phantom image contained 24 diamond-shaped objects oriented along four directions $\left(0^{\circ}, 30^{\circ}, 60^{\circ}\right.$, and $\left.90^{\circ}\right)$ and six scales, as shown in Fig. 15(a). After applying our algorithm to this image, the borders of these objects at each scale were clearly enhanced. The phantom objects in the last two rows of the image, are very close to the size of microcalcifications. This demonstrates the ability of the algorithm to detect typical microcalcification clusters in digital mammograms, without artifacts.

Three examples of malignant lesions with distinct radiographic signs of cancer were processed. The images shown are of matrix size $512 \times 512$. For each case, both global and ROI's are shown along with a corresponding enhanced ROI.

1) Microcalcifications: Fig. 16 shows a sample mammogram with microcalcification clusters. The original mammogram is shown in Fig. 16(a). Fig. 16(b) shows an original suspicious area out of the mammogram. After enhancement, clusters of calcifications clearly appear in the center of the image.

2) Stellate Lesions: A mammogram with a stellate lesion is shown in Fig. 17(a). Fig. 17(b) presents an original digital radiograph with a partially obscured irregular mass in the center of the image matrix. After applying our algorithm, the enhanced image makes obvious spiculated lesions around 


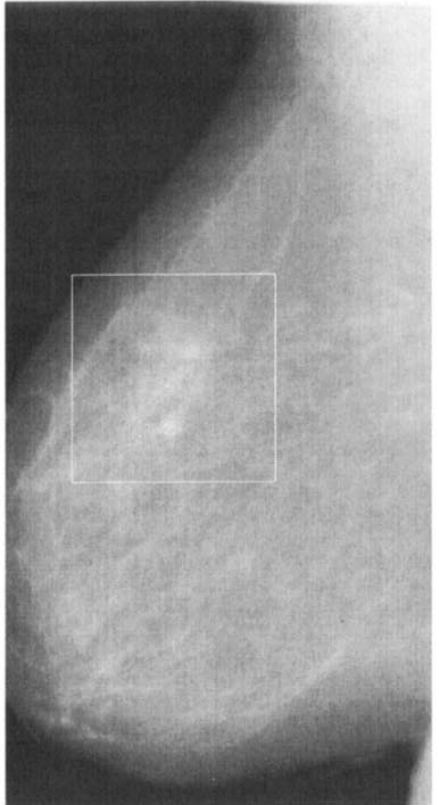

(a)

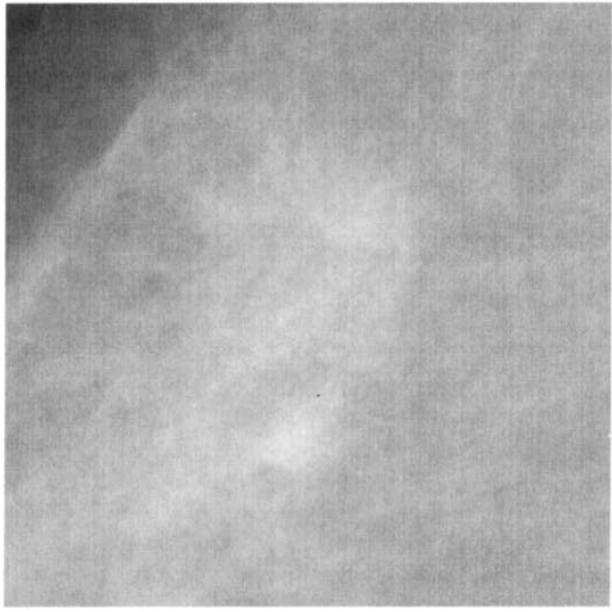

(b)

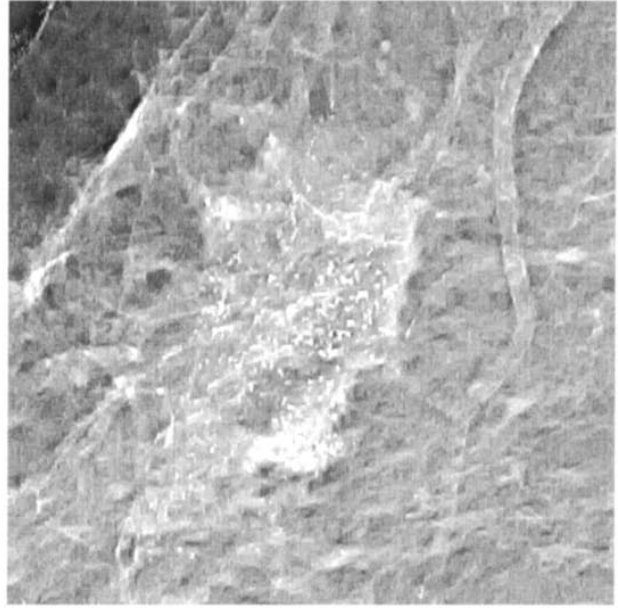

(c)

Fig. 16. Mammogram with calcifications. (a) Original mammogram. (b) ROI image. (c) Enhanced ROI image.

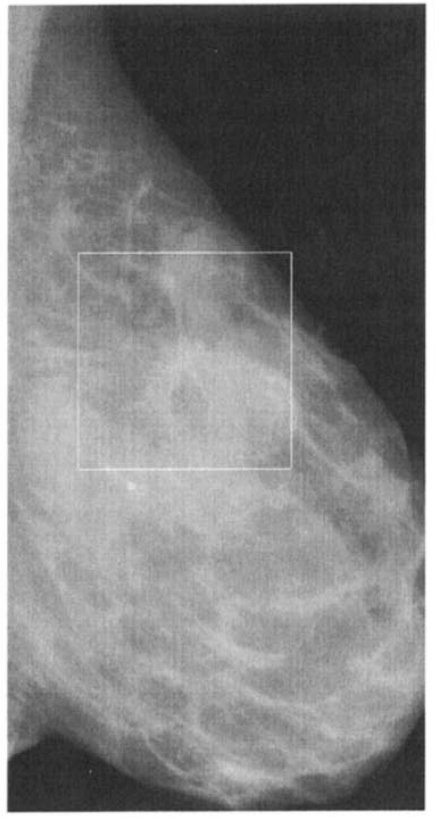

(a)
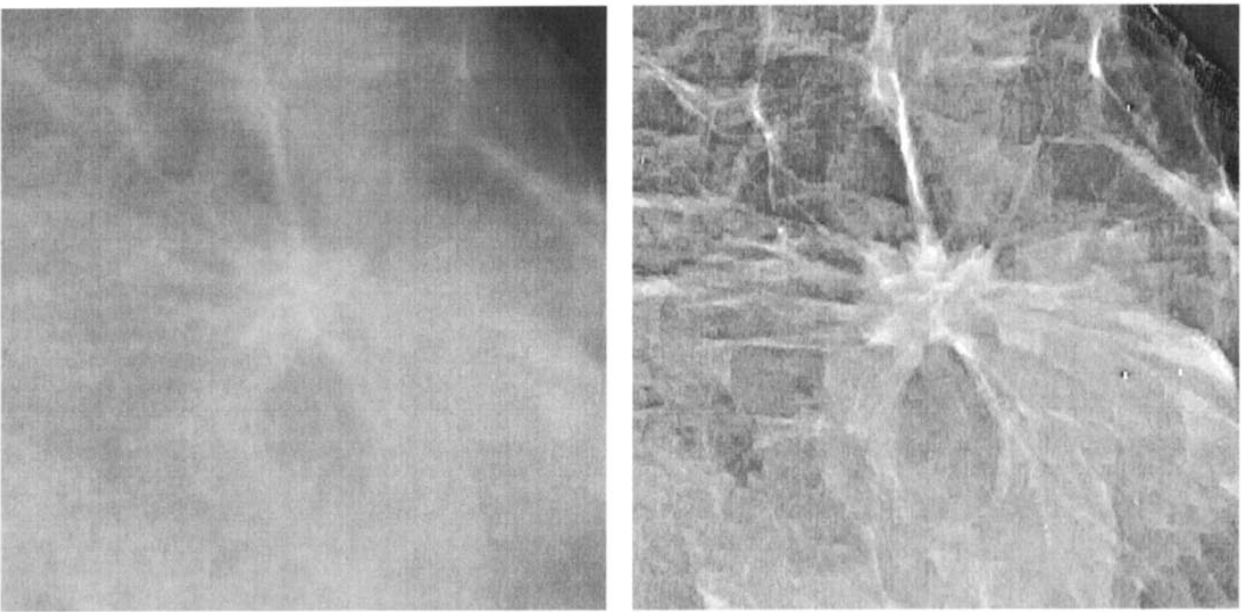

(b)

(c)

Fig. 17. Mammogram with a stellate lesion. (a) Original mammogram. (b) ROI image. (c) Enhanced ROI image.

the mass. The mass itself was also enhanced, as shown in Fig. 17(c). These clear spiculated patterns suggest to radiologists that this mass is more likely malignant than benign.

3) Masses: A mammogram including a mass tumor is shown in Fig. 18. The craniocaudal view of the left breast shown in Fig. 18(b) contains an irregular spiculated mass in retroglandular fat. The enhanced ROI shown in Fig. 18(c) better delineates the margins of the mass in this surround.

4) Quantitative Performance: To validate our enhancement techniques, three mathematical models of phantoms were constructed. The models included features of interest in mam- mographic imaging, such as masses, microcalcifications, and spicular lesions, as shown in Fig. 19. Each phantom was blended into a normal mammogram to form an experimental database. Note that the locations of these mathematical models are distinct within each image. We constructed 20 cases in our testing database. The test images were of matrix size $1024 \times 1024$.

Many techniques of contrast manipulation and modification have been developed within the field of digital image processing. However, the measurement and evaluation of contrast and contrast changes in arbitrary images are not uniquely defined 


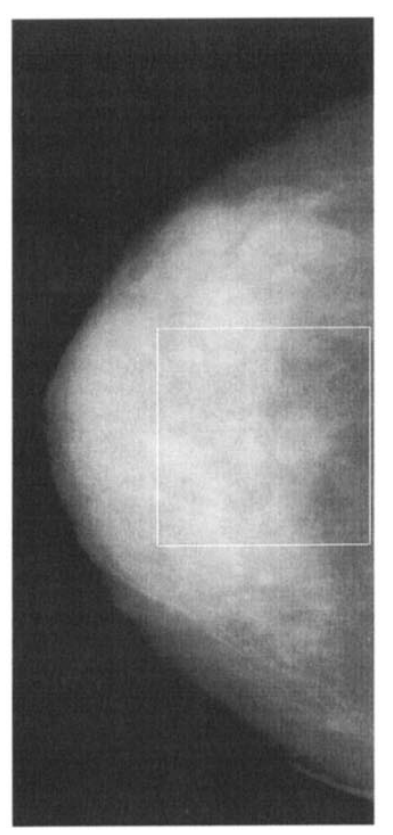

(a)

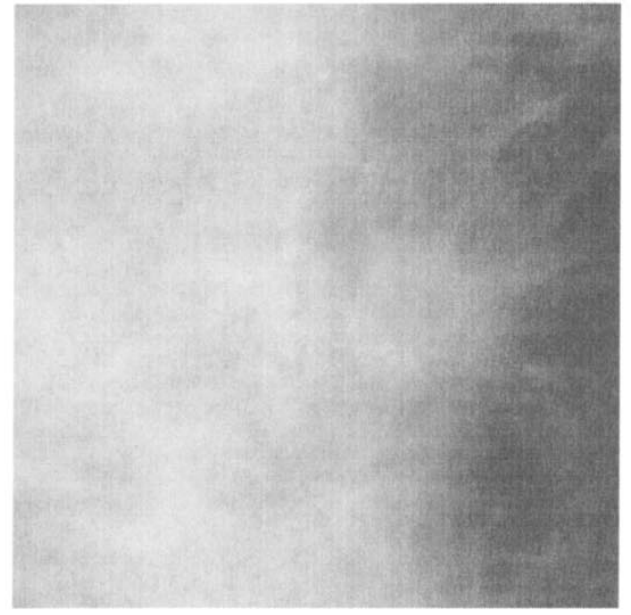

(b)

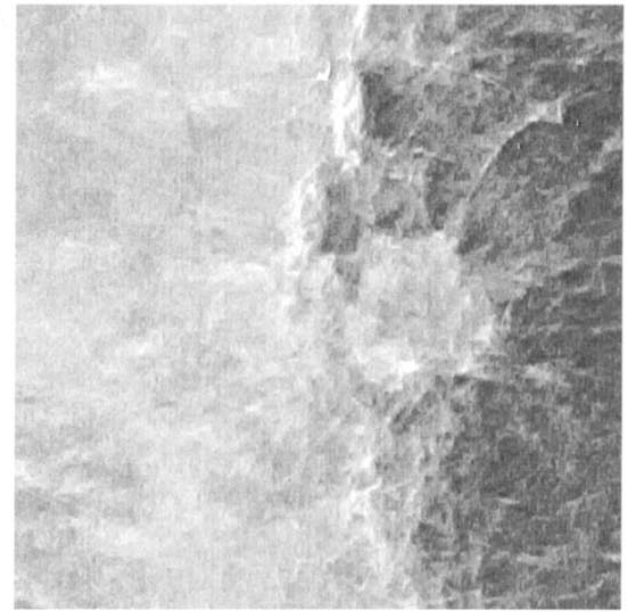

(c)

Fig. 18. Mammogram with a mass. (a) Original mammogram. (b) ROI image. (c) Enhanced ROI image.

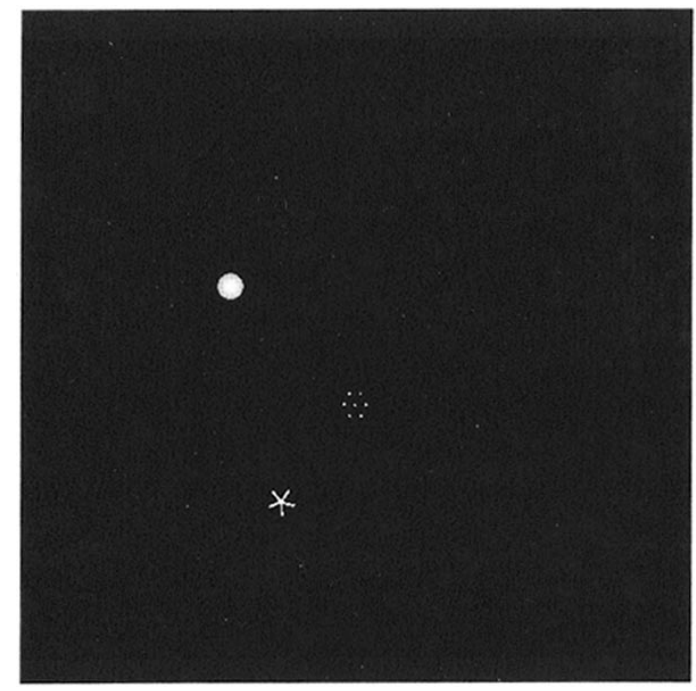

(a)

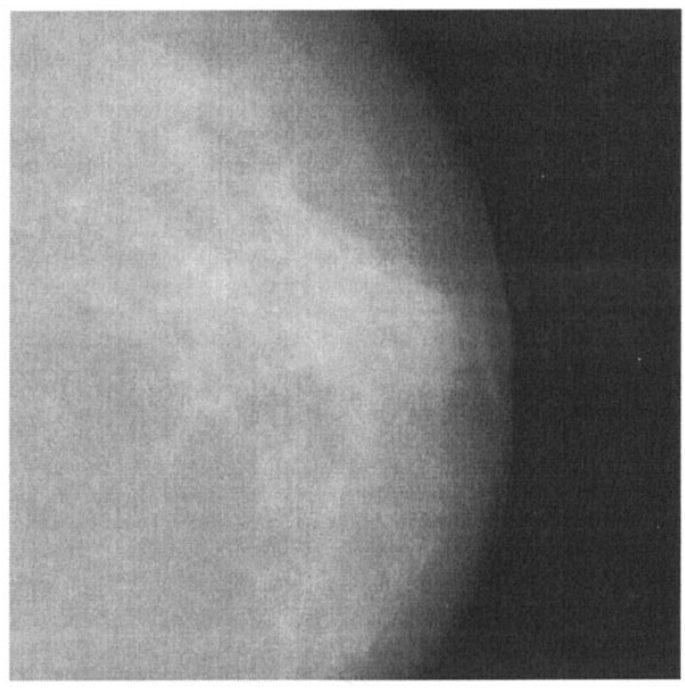

(b)

Fig. 19. (a) Mathematical phantoms of mammographic features. (b) A blended sample image.

in the literature [8], [25]. In this paper, we adopt a definition introduced by Morrow et al. [23]

$$
C_{t}=\frac{B_{0}-B}{B_{0}+B}
$$

where $B$ is the mean gray-level value of a particular object in an image (foreground) and $B_{0}$ is the mean gray-level value of a surrounding region (background).

Based on this definition, we applied the proposed algorithm to each blended image and computed distinct contrast values. Three mathematical models of phantoms were constructed of size $40 \times 40$. Pixel features were considered either foreground or background. We used a quantitative measure of contrast improvement, previously defined by a contrast improvement index (CII) [17], [16]

$$
C I I=\frac{C_{t}^{\text {processed }}}{C_{t}^{\text {original }}}
$$

where $C_{t}^{\text {processed }}$ and $C_{t}^{\text {original }}$ are the contrast values for a region of interest in the processed and original images, respectively.

We compared our results with those of four existing contrast enhancement techniques: contrast stretching (CS), unsharp masking (UM), histogram equalization (HE), and treestructured filters (TSF's) [28], [27]. Fig. 20 shows some 


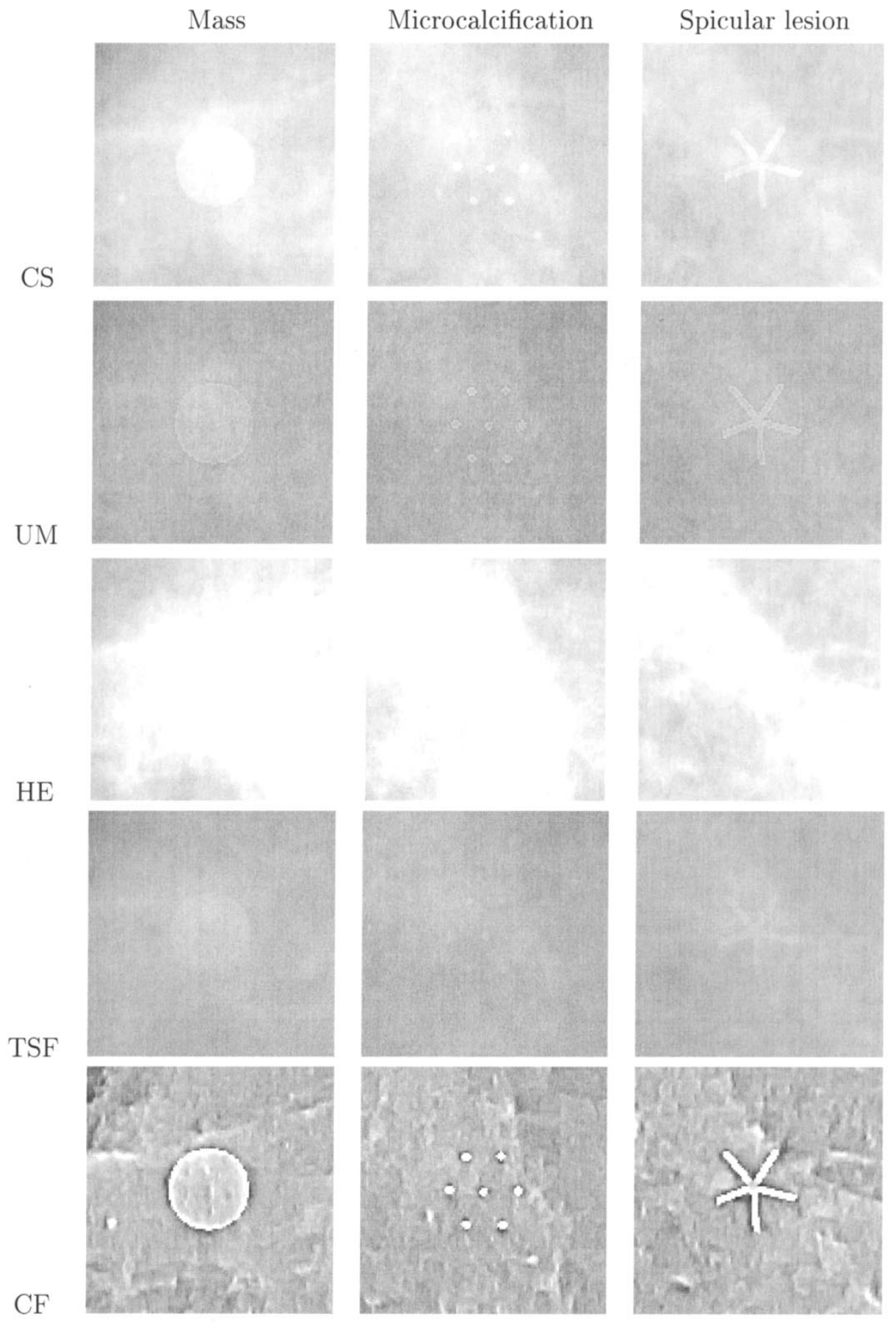

Fig. 20. Sample results of five different methods.

processed results of the ROI's from a sample using five different methods. Results of the comparison are summarized in Tables I-III. From these results, we observe that the values of CII depend on the image itself, the types of features, and the surrounding context of the features. The methods of CS and UM consistently showed little improvement over the original image, while HE exhibited inconsistent results. Finally, the TSF method showed no quantitative improvement. Our algorithm consistently outperformed each of these methods. Note that masses have larger regions than microcalcifications and spicular lesions. However, since our method emphasized edge features, the mean values of the masses were consequently less improved.

\section{CONCLUSION}

An enhancement algorithm relying on multiscale wavelet analysis and extracted oriented information at each scale of analysis was investigated. The evolution of wavelet coefficients across scales characterized well the local shape of irregular structures. Using oriented information to detect features of an image appears to be a promising approach for enhancing complex structures and subtle tissues of the 
TABLE I

CONTRAST IMPROVEMENT INDEX FOR MASSES ObtaINEd by CS, UM, HE, TSF, and CF Methods

\begin{tabular}{c||c|c|c|c|c}
\hline Case & CS & UM & IIE & TSF & CF \\
\hline \hline 1 & 1.2321 & 1.1538 & 0.3934 & 0.9990 & 3.4121 \\
2 & 1.2720 & 1.1627 & 0.5430 & 0.9996 & 3.5911 \\
3 & 1.2453 & 1.1370 & 1.1606 & 0.9993 & 4.1997 \\
4 & 1.2218 & 1.1109 & 0.6830 & 0.9989 & 3.5410 \\
5 & 1.2313 & 1.1125 & 0.2755 & 1.0004 & 3.7025 \\
6 & 1.2590 & 1.2214 & 2.3413 & 0.9978 & 4.2358 \\
7 & 1.2458 & 1.2080 & 3.0524 & 0.9995 & 4.9465 \\
8 & 1.2374 & 1.0895 & 0.9045 & 0.9991 & 4.2415 \\
9 & 1.3271 & 1.1393 & 1.7784 & 0.9994 & 6.1136 \\
10 & 1.2236 & 1.1569 & 0.8641 & 0.9989 & 3.9763 \\
11 & 1.2622 & 1.0887 & 1.2967 & 0.9993 & 3.6320 \\
12 & 1.2485 & 1.1150 & 3.7718 & 0.9998 & 5.9985 \\
13 & 1.2543 & 1.1119 & 1.1636 & 0.9992 & 3.1344 \\
14 & 1.2742 & 1.1809 & 2.5198 & 0.9984 & 4.7512 \\
15 & 1.2332 & 1.1084 & 2.0114 & 0.9994 & 3.8008 \\
16 & 1.2316 & 1.1533 & 0.9142 & 0.9988 & 4.1431 \\
17 & 1.2071 & 1.1520 & 0.1207 & 0.9994 & 3.0553 \\
18 & 1.2233 & 1.1074 & 2.4443 & 0.9999 & 3.9690 \\
19 & 1.2275 & 1.1273 & 2.0329 & 0.9999 & 3.1121 \\
20 & 1.2614 & 1.1678 & 1.9330 & 0.9990 & 3.8542 \\
\hline
\end{tabular}

TABLE II

CONTRAST IMPROVEMENT INDEX FOR MicROCALCIFICATIONS OBTAINED By CS, UM, HE, TSF, AND CF METHODS

\begin{tabular}{c||c|c|c|c|c}
\hline Case & CS & LM & HF & TSF & CF \\
\hline \hline 1 & 1.2510 & 2.0225 & 3.0738 & 0.9850 & 8.0812 \\
2 & 1.2855 & 1.9996 & 1.5135 & 0.9957 & 8.8415 \\
3 & 1.2811 & 1.9945 & 2.1976 & 0.9929 & 9.0558 \\
4 & 1.2204 & 1.9953 & 0.6681 & 0.9953 & 6.1101 \\
5 & 1.2322 & 1.9159 & 0.3781 & 0.9966 & 6.5205 \\
6 & 1.2703 & 1.9731 & 1.3389 & 0.9940 & 7.8585 \\
7 & 1.2335 & 1.9508 & 0.6793 & 0.9926 & 6.5333 \\
8 & 1.2394 & 2.0097 & 1.1124 & 0.9957 & 7.7731 \\
9 & 1.2636 & 1.7277 & 1.7397 & 0.9916 & 6.5394 \\
10 & 1.2280 & 2.1050 & 0.8854 & 0.9901 & 5.8531 \\
11 & 1.3195 & 1.7763 & 1.5760 & 0.9947 & 6.1981 \\
12 & 1.2354 & 1.9701 & 2.0045 & 0.9966 & 6.8729 \\
13 & 1.2938 & 2.1740 & 3.4047 & 0.9852 & 6.1026 \\
14 & 1.2401 & 1.9956 & 0.1757 & 0.9845 & 7.3612 \\
15 & 1.2840 & 2.1781 & 1.0409 & 0.9979 & 5.8868 \\
16 & 1.2452 & 1.9345 & 2.1372 & 0.9894 & 6.6954 \\
17 & 1.2438 & 1.9107 & 0.9802 & 0.9904 & 6.1960 \\
18 & 1.2246 & 1.9772 & 2.3434 & 0.9920 & 6.5619 \\
19 & 1.2299 & 1.9302 & 2.1773 & 0.9907 & 6.3651 \\
20 & 1.2308 & 1.9459 & 1.0755 & 0.9970 & 5.9010 \\
\hline
\end{tabular}

breast. Steerable filters which can be rotated at arbitrary orientations reliably found visual cues within each spatialfrequency sub-band of an image. Coherence measure and dominant orientation clearly improved discrimination of features from complex surrounding tissue and structure in dense mammograms.

We examined existing and previous multiscale enhancement approaches [15], [17], [30] which attempted to enhance an image by detecting edges. Unfortunately, traditional edge detection algorithms cannot distinguish between authentic edges and phantom edges. In contrast, the $\mathrm{CF}$ algorithm described here relied upon oriented energy functions to identify edges more precisely. Coherence measures removed noise and gave
TABLE III

CONTRAST IMPROVEMENT INDEX FOR SPICULAR LESIONS OBtaINED by CS, UM, HE, TSF, AND CF Methods

\begin{tabular}{c||c|c|c|c|c}
\hline Case & CS & UM & HE & TSF & $\overline{\text { CF }}$ \\
\hline \hline 1 & 1.2576 & 1.5373 & 3.5718 & 0.9981 & 6.4958 \\
2 & 1.3016 & 1.6564 & 1.5741 & 0.9983 & 8.9896 \\
3 & 1.2709 & 1.6748 & 1.5917 & 0.9968 & 9.0420 \\
4 & 1.2647 & 2.1918 & 2.6259 & 0.9934 & 14.8793 \\
5 & 1.2397 & 1.5258 & 1.0526 & 0.9970 & 6.4616 \\
6 & 1.2508 & 1.5366 & 1.5741 & 0.9959 & 6.4331 \\
7 & 1.2432 & 1.5318 & 2.1020 & 0.9990 & 6.4341 \\
8 & 1.2837 & 1.7600 & 1.1114 & 0.9956 & 11.1680 \\
9 & 1.3500 & 1.6194 & 1.0969 & 0.9963 & 10.5974 \\
10 & 1.2203 & 1.6627 & 0.6285 & 0.9978 & 7.1692 \\
11 & 1.2498 & 1.6466 & 1.7991 & 0.9969 & 7.1298 \\
12 & 1.2654 & 1.6334 & 2.1535 & 0.9970 & 8.9059 \\
13 & 1.2835 & 1.6544 & 1.0977 & 0.9972 & 5.4763 \\
14 & 1.2495 & 1.6130 & 0.6353 & 0.9971 & 7.8101 \\
15 & 1.2860 & 1.7091 & 1.0337 & 0.9934 & 5.3742 \\
16 & 1.2332 & 1.6502 & 1.1970 & 0.9975 & 7.2288 \\
17 & 1.3066 & 1.4105 & 1.6293 & 0.9999 & 6.3881 \\
18 & 1.2135 & 1.5815 & 0.8470 & 0.9989 & 6.2900 \\
19 & 1.2527 & 1.5995 & 3.1518 & 0.9984 & 7.1808 \\
20 & 1.2478 & 1.6107 & 3.3187 & 0.9976 & 6.1226 \\
\hline
\end{tabular}

a more reliable basis for contrast enhancement. Phase information provided an adjustment for the correction of wavelet coefficients. These modifications resulted in an enhancement more familiar to clinical radiologists. Artifact-free enhanced images, as shown here, can be a powerful tool for visualizing subtle features of importance to mammography.

\section{REFERENCES}

[1] H. G. Barrow and J. M. Tenenbaum, "Recovering intrinsic scene characteristics from images," in A. Hanson and E. Riseman, Eds., Computer Vision Systems. New York: Academic, 1978, pp. 3-26.

[2] K. R. Castleman, Digital Image Processing. Upper Saddle River, NJ: Prentice-Hall, 1996.

[3] C. M. Chang, Coherence of Multiscale Features for Enhancement of Digital Mammograms, Ph.D. dissertation, Univ. Florida, Gainesville, 1997

[4] C. M. Chang and A. Laine, "Enhancement of mammogram from oriented information," in Proc. IEEE Int. Conf. Image Processing, Santa Barbara, CA, 1997, vol. 3, pp. 524-527.

[5] R. L. Egan, Breast Imaging. Philadelphia, PA: W. B. Saunders, 1988.

[6] W. T. Freeman and E. H. Adelson, "The design and use of steerable filters," IEEE Trans. Pattern Anal. Machine Intell., vol. 13, pp. 891-906, Sept. 1991.

[7] S. L. Hahn, Hilbert Transforms in Signal Processing. Norwood, MA: Artech, 1996.

[8] E. L. Hall, Computer Image Processing and Recognition. New York: Academic, 1979.

[9] M. Holschneider, R. Kronland-Martinet, J. Morlet, and $\mathrm{Ph}$. Tchamitchian, "A real-time algorithm for signal analysis with the help of the wavelet transform," in J. M. Combes, A. Grossman, and Ph. Tchamitchian, Eds., Wavelets: Time-frequency Methods and Phase Space. Berlin, Germany: Springer-Verlag, 1990, pp. 286-304.

[10] W. P. Kegelmeyer Jr., J. M. Pruneda, P. D. Bourland, A. Hillis, M. W. Riggs, and M. L. Nipper, "Computer-aided mammographic screening for spiculated lesions," Radiology, vol. 191, no. 2, pp. 331-337, 1994

[11] N. Karassemeijer and G. M. te Brake, "Detection of stellate distortions in mammograms," IEEE Trans. Med. Imag., vol. 15, no. 5, pp. 611-619, 1996.

[12] M. Kass and A. Witkin, "Analyzing oriented patterns," Computer Vision, Graphics, and Image Processing, vol. 37, no. 3, pp. 362-385, 1987.

[13] J. J. Koenderink and A. J. van Doorn, "Generic neighborhood operators," IEEE Trans. Pattern Anal. Machine Intell., vol. 14, pp. 597-605, June 1992

[14] A. Laine and C. M. Chang, "De-noising via wavelet transforms using steerable filters," in IEEE Int. Symp. Circuits and Systems, 1995, Seattle, 
WA, vol. 3, pp. 1956-1959.

[15] A. Laine and S. Song, "Multiscale wavelet representations for mammographic feature analysis," in Mathematical Methods in Medical Imaging, Proc. SPIE, San Diego, CA, 1992, vol. 1768, pp. 306-316.

[16] A. F. Laine, J. Fan, and W. Yang, "Wavelets for contrast enhancement of digital mammography," IEEE Eng. Medicine Biol., vol. 14, no. 5, pp. 536-550, 1995.

[17] A. F. Laine, S. Schuler, J. Fan, and W. Huda, "Mammographic feature enhancement by multiscale analysis," IEEE Trans. Med. Imag., vol. 13, pp. 725-740, Apr. 1994.

[18] L. Li, W. Qian, and L. P. Clark, "X-ray medical image processing using directional wavelet transform," in Proc. IEEE Int. Conf. Acoustics, Speech, and Signal Processing, 1996, Atlanta, GA, vol. 4, pp. 2251-2254.

[19] S. Mallat and S. Zhong, "Characterization of signals from multiscale edges," IEEE Trans. Pattern Anal. Machine Intell., vol. 14, pp. 710-732, July 1992

[20] D. Marr, Vision. San Francisco, CA: Freeman, 1982.

[21] R. McLelland, "Stellate lesions of the breast," Recent Results in Cancer Research, vol. 19, pp. 24-27, 1990.

[22] M. C. Morrone and R. A. Owens, "Feature detection from local energy," Pattern Recognit. Lett., vol. 6, no. 5, pp. 303-313, 1987.

[23] W. M. Morrow, R. B. Paranjape, R. M. Rangayyan, and J. E. Leo Desautels, "Region-based contrast enhancement of mammograms," IEEE Trans. Med. Imag., vol. 11, pp. 392-406, Mar. 1992.

[24] R. Owens, S. Venkatesh, and J. Ross, "Edge detection is a projection," Pattern Recognit. Lett., vol. 9, no. 4, pp. 233-244, 1989.

[25] E. Peli, "Contrast in complex images," J. Opt. Soc. Amer. A, vol. 7, no. 10, pp. 2032-2040, 1990.

[26] P. Perona, "Deformable kernels for early vision," IEEE Trans. Pattern Anal. Machine Intell., vol. 17, pp. 488-499, May 1995.

[27] W. Qian, L. P. Clarke, M. Kallergi, and R. A. Clark, "Tree-structured nonlinear filters in digital mammography," IEEE Trans. Med. Imag., vol. 13, pp. 25-36, Jan. 1994.

[28] W. Qian, L. P. Clarke, M. Kallergi, H.-D. Li, R. Velthuizen, R. A Clark, and M. L. Silbiger, "Tree-structured nonlinear filter and wavelet transform for microcalcification segmentation in mammography," in Proc. SPIE Biomedical Image Processing and Biomedical Visualization, San Jose, CA, vol. 1905, pp. 509-520, 1993.

[29] A. R. Rao and B. G. Schunck, "Computing oriented texture fields," in Proc. IEEE Computer Soc. Conf. Computer Vision and Pattern Recognition, San Diego, CA, 1989, pp. 61-68.

[30] W. B. Richardson Jr., "Nonlinear filtering and multiscale texture discrimination for mammograms," in Proc. SPIE Mathematical Methods in Medical Imaging, San Diego, CA, vol. 1768, pp. 293-305, 1992.

[31] M. J. Shensa, "The discrete wavelet transform: Wedding the à trous and Mallat alogrithms," IEEE Trans. Signal Processing, vol. 40, pp. 2464-2482, Oct. 1992

[32] G. Strang, "Wavelets and dilation equations: A brief introduction," SIAM Rev., vol. 31, no. 4, pp. 614-627, 1989.
[33] Y. Xing, W. Huda, A. Laine, J. Fan, B. Steinbach, and J. Honeyman, "Comparison of a dyadic wavelet image enhancement algorithm with unsharp masking and median filtering," in Proc. SPIE Medical Imaging: Image Processing, San Diego, CA, vol. 2434, pp. 718-729, 1995.

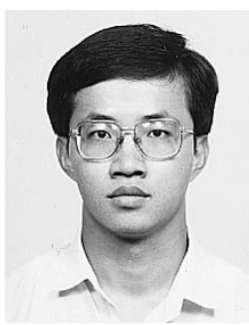

Chun-Ming Chang was born in Changhua, Taiwan. He received the B.S. degree from the National Cheng Kung University, Taiwan, in 1985 and the M.S. degree from the National Tsing Hua University, Taiwan, in 1987, both in electrical engineering. $\mathrm{He}$ received the Ph.D. degree in electrical and computer engineering from the University of Florida, Gainesville, in 1997. His dissertation focused on the application of multiscale representations for medical image analysis.

From 1989 to 1991 , he was a Lecturer in the Department of Electronic Engineering of Kwang Wu College, Taipei, Taiwan. He joined VR Telecom Corporation, a telecommunication company located in Pittsburgh, PA, as a Senior Technical Staff Member in 1998. His research interests include image processing, computer graphics, robotics, artificial intelligence, wavelet analysis, and video compression.

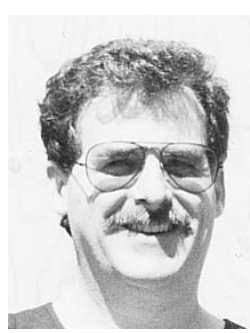

Andrew Laine received the B.S. degree from Cornell University, Ithaca, NY, and the M.Sc. and D.Sc. degrees from Washington University, St. Louis, MO, in 1983 and 1989, respectively.

$\mathrm{He}$ is an Associate Professor of Biomedical Engineering and Radiology in the Department of Biomedical Engineering, Columbia University, New York, NY. His research interests include methods of multiresolution analysis applied to problems in medical imaging, computer vision, pattern recognition, graphics, and applied mathematics. He served as a Guest Editor for the Journal of Mathematical Imaging and Vision special issue on "Wavelet Theory and Application" in March 1993 and edited the book Wavelet Theory and Application (Norwell, MA: Kluwer, 1993). He chaired the annual SPIE conference on "Wavelet Applications in Signal and Image Processing" from 1993 to 1999.

Dr. Laine is a member of the IEEE Computer Society and the IEEE Engineering in Medicine and Biology Society (IEEE-EMBS). He served as an Associate Editor of IEEE Transactions on IMAge Processing (1996-1998). $\mathrm{He}$ is a member of the Editorial Board of the book series Emerging Technologies in Biomedical Engineering, sponsored by the IEEE-EMBS. 